\title{
Supra-galactic colour patterns in globular cluster systems
}

\author{
Juan C. Forte, ${ }^{1,2 \star}$ \\ ${ }^{1}$ Consejo Nacional de Investigaciones Científicas y Técnicas (CONICET), Argentina \\ ${ }^{2}$ Planetario 'Galileo Galilei', Ministerio de Modernización, Innovación y Tecnlogía, Ciudad Autónoma de Buenos Aires, Argentina
}

Accepted:March 11, 2017; Received: March 5, 2017; in original form: January 29, 2017

\begin{abstract}
An analysis of globular cluster systems associated with galaxies included in the Virgo and Fornax HST-Advanced Camera Surveys reveals distinct $(g-z)$ colour modulation patterns. These features appear on composite samples of globular clusters and, most evidently, in galaxies with absolute magnitudes $M_{g}$ in the range from -20.2 to -19.2. These colour modulations are also detectable on some samples of globular clusters in the central galaxies NGC 1399 and NGC 4486 (and confirmed on data sets obtained with different instruments and photometric systems), as well as in other bright galaxies in these clusters. After discarding field contamination, photometric errors and statistical effects, we conclude that these supra-galactic colour patterns are real and reflect some previously unknown characteristic. These features suggest that the globular cluster formation process was not entirely stochastic but included a fraction of clusters that formed in a rather synchronized fashion over large spatial scales, and in a tentative time lapse of about 1.5 Gy at redshifts $z$ between 2 and 4. We speculate that the putative mechanism leading to that synchronism may be associated with large scale feedback effects connected with violent star forming events and/or with super massive black holes.
\end{abstract}

Key words: galaxies: star clusters - ISM: jets and outflows.

\section{INTRODUCTION}

Globular clusters (GCs), both as individuals and as members of systems $(G C S)$ in different galaxies, can be genuinely considered as crucial and ever-surprising Rosetta stones. The astrophysical information they contain, however, has not been completely decoded yet. Among other issues, the presence of multiple stellar populations (e.g. Milone et al. 2017) within a given cluster is a relatively new challenge that adds to other historical questions: How do they form and why this process is not evident in low $z$ galaxies, except in particular cases (e.g. galaxy mergers), where some objects seem to resemble the young counterparts of $G C s$.

A prominent characteristic of $G C S$ in massive galaxies is that their globular cluster colour distributions (GCCDs in what follows) are frequently "bimodal", i.e., these distributions are dominated by two ("blue" and "red") cluster families. This feature is known since the mid 90's and an account of the contributions that led to its discovery is given in Brodie \& Strader (2006). As shown by Peng et al. (2006), the presence of "bimodality" depends on the absolute magnitude of a given galaxy. Blue globulars are present in practically all galaxies while the red $G C$ sub-population becomes more important as the galaxy brightness increases.

* E-mail: planeta.jcf@gmail.com
In general, blue GCs exhibit shallow spatial distributions and seem connected with dark matter halos. In turn, red GCs show more concentrated distributions, that are comparable with the galaxy surface brightness, at least in the inner regions (Faifer et al. 2011; Forbes et al. 2012, 2016; Forte et al. 2012). Recent works (D'Abrusco, Fabbiano \& Zezas 2015) indicate the presence of complex structures on top of those overall spatial patterns.

In a broad sense, bimodality seems just to reflect the halo-bulge structures common to most massive galaxies. The description of $G C$ populations in terms of two components, however, is probably an over simplification of the general situation. An updated and thorough summary of the arguments that justify an effort to advance "beyond bimodality" is given by Harris et al. (2017).

This work presents an exploratory attempt in that direction. The underlying issue is if, besides the usual decoding of the GCCDs in terms of age, chemical abundance, and spatial distribution, there might be other (more subtle) features, providing a different kind of information in these distributions.

An interesting aspect, in this last context, is the possible detectability of relics of energetic events on the stellar populations in the early Universe that, later on, survived until today. As frequently stated, globular clusters appear as 
remarkable survivors born in those ages (e.g. Forbes et al. 2015; Vanzella et al. 2016) and could be a suitable workbench to test that possibility.

Observational evidence in the sense that powerful nuclear outflows may have been common, leaving imprints not only in the host galaxy but also on larger spatial scales, is reviewed in Fabian (2012). In this scenario, nuclear super massive black holes $(S M B H)$ may have played a significant role.

The importance of both star formation and $A G N s$ on the parameters that characterize the interstellar medium, and have an impact on that process, has become clear in recent years as shown by galaxy formation models (e.g Vogelsberger et al. 2014).

The literature includes some previous works that have discussed a possible connection between high energy phenomena and GCs. In this frame, the remarkable homogeneity of the (almost) ever present blue (halo) GCs, suggests that their formation may have been regulated by large spatial scale events like, for example, the re-ionization of the Universe (e.g. Cen 2001).

The usual procedure to study the GCCDs along the years has been based on discrete and/or "generalized" (smoothed) histogram versions of these distributions.

In this paper we present a somewhat different approach. Instead of analysing $G C s$ as members of a single galaxy, we work on composite samples of clusters that belong to galaxies within a given sampling window, defined in the galaxy absolute magnitude $M_{g}$ vs. $(g-z)$ colour plane.

This procedure will presumably erase the "personality" of a given GCS but might enhance the presence of systemic features, if any, common to these galaxies. For example, $G C$ colour patterns that could arise as a result of old feedback phenomena.

The core of the analysis is the ACS photometry of $G C s$ associated with galaxies in the the Virgo and Fornax clusters presented, respectively, by Jordán et al. (2009) and Jordán et al. (2015). In the case of the central and massive galaxies $N G C 1399$ and $N G C$ 4486, we revisit the GCs photometry given in Forte, Faifer \& Geisler (2007) and Forte et al. (2013).

The structure of the paper is as follows: Section 2 gives a summary of the characteristics of the photometric data, assumed distance moduli and interstellar reddening; Section 3 presents an overall view of the $G C$ colour distributions for galaxies in Virgo and Fornax, both in the form of discrete and generalized histograms; the procedure adopted for the recognition of eventual patterns in the $G C$ colour distributions is described in Section 4; in Section 5, we present the analysis of $G C s$ in Virgo and Fornax galaxies with $M_{g}=$ -20.2 to -19.2 ; Section 6 describes an attempt to identify the colour patterns in $G C s$ associated with the central galaxies $N G C 1399$ and NGC 4486; a tentative decomposition of the $G C C D$ in terms of discrete mono-colour populations is described in Section 7; Finally, Sections 8 and 9 present a discussion of the results and the conclusions, respectively.

\section{DATA SOURCES}

The details concerning the $G C$ photometry in the Virgo and Fornax ACSs have been extensively discussed in
Jordán et al. (2009) and Jordán et al. (2015). The galaxy morphology in these surveys is dominated by elliptical and lenticular galaxies.

In what follows, the $G C(g-z)$ colours are corrected for interstellar reddening and taken from Table 4 and Table 2, for Virgo and Fornax GCs, respectively, in those papers. Aiming at an homogeneous comparison between the GCS in Virgo and Fornax, we adopted upper galactocentric radii of 110 " and 90.6 ", respectively. These radii correspond to a galactocentric radius of $8.8 \mathrm{Kpc}$ after adopting the $S B F$ distance moduli given by Blakeslee et al. (2009).

We also set an apparent magnitude $g=25.0$ (about a magnitude above the $A C S$ limiting magnitude) as the faintest limit of the analysis. This limit guarantees both a high areal completeness level and mean colour errors of $\approx$ $0.07 \mathrm{mag}$.

For old GCs (i.e. $12 G y)$ we expect a $(g-z) o$ colour range from 0.75 to 1.55 (see, for example, Forte et al. 2013). In this work we adopt a wider colour window $((g-z) o$ from 0.50 to 1.80$)$, aiming at including eventually younger or older clusters. The absolute $M_{g}$ magnitude vs $(g-z)$ colour diagram for 88 galaxies in Virgo and 42 galaxies in Fornax is displayed in Fig. 1

The $M_{g}$ magnitudes were derived from the $B$ band photometry by Binggeli, Sandage \& Tarenghi (1984) and Ferguson (1989), the relation between $g$ and $B$ magnitudes given by Chonis \& Gaskell (2008), and the $(g-r)$ vs $(g-z)$ relation for galaxy colours we derived from Chen et al. (2010).

Both the galaxy magnitudes and colours were corrected adopting the reddening maps presented in Schlafly \& Finkbeiner (2011). From these maps, the mean colour excess for Virgo is $E_{(B-V)}=0.03 \pm 0.01$ and $E_{(B-V)}=0.01 \pm 0.005$ for Fornax. The formal $\mathrm{rms}$ errors of both values are very low but, in fact, the rms of the original infra-red emission vs. colour excess map calibration is considerably larger, and close to $\pm 0.03 \mathrm{mag}$.

Giant galaxies lie above the horizontal line at $M_{g}=-$ 20.2 in Fig. 1. For these objects the areal coverage of the $A C S s$ is relatively low (see Fig.4 in Forte et al. 2014). Some of these objects, given their status as central and dominant galaxies, are discussed in Section 6 .

For the particular analysis of the central galaxies $N G C 4486$ and $N G C$ 1399, we re-analyse the photometry presented by Forte et al. (2007). These homogeneous ground based observations were obtained with the the Mayall and Blanco 4-m telescopes at KPNO and CTIO, adopting the $\left(C-T_{1}\right)$ colour index defined with pass bands of the Washington photometric system. In the case of $N G C 4486$, we also revisit the Gemini-GMOS photometry for some 500 GCs located in a peripheral field $\left(\approx 2.5^{\prime}\right.$ to the south of the galaxy centre).

\section{DISCRETE AND SMOOTHED GCCD IN VIRGO AND FORNAX GALAXIES}

The usual procedure in the analysis of the $G C C D$ is the adoption of discrete colour bin histograms. The size of these bins is commonly set on an assessment of the colour errors (e.g. adopting a bin size comparable to the photometric errors), or aiming at decreasing the statistical noise of the 


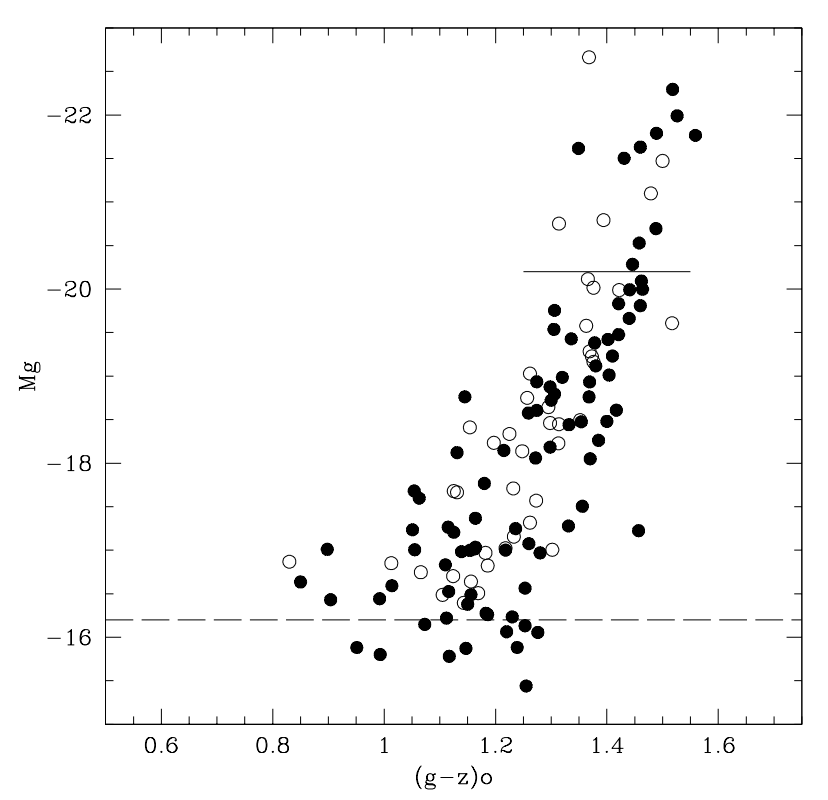

Figure 1. Absolute $M_{g}$ magnitude vs. (g-z)o colours for Virgo (filled circles) and Fornax (open circles) ACS galaxies. Galaxies brighter than $M_{g}=-20.2$ (solid line) are considered as "giants". The dashed line at $M_{g}=-16.2$ defines the faint boundary of the analysis.

data through variable bin size histograms, as in Peng et al. (2006).

An alternative approach are smoothed colour distributions that can be derived by convolving the colour data with, for example, Gaussian kernels whose standard deviation parameters are also set in terms of the photometric errors.

Needless to say that this smoothing decreases the resolution in colour even more, as it adds to the "blurring" already introduced by photometric errors.

In both cases (i.e, discrete bins or smoothed data), the central issue is the compromise between colour resolution and the signal to statistical noise ratio. In the search for systemic features, however, the smoothed GCCDs are more sensitive to data "clumpines" and allow the identification of subtle structures, provided the adoption of a proper value for the smoothing kernel.

In this paper we adopt both representations of the GCCDs. The bin size of discrete histograms, and the Gaussian kernel parameter $\left(\sigma_{(g-z)}\right)$ were tuned up in such a way that, the eventual peaks seen both in the discrete bins and in the smoothed data, do not differ by more than 0.02 mags.

Experimenting with these parameters on the ACSs photometric data, we find that, $0.04 \mathrm{mag}$ bins and a $\sigma_{(g-z)}=0.015 \mathrm{mag}$ Gaussian kernel, meet that condition. Smoothing kernels larger than $\approx 0.04$ mag., practically erase all the substructure present in the GCCDs and just leave, depending the case, broad uni modal or bimodal distributions.

Fig. 2 and Fig. 3 display the composite $G C C D$, in both formats, for $G C s$ brighter than $M_{g}=-6.5$, in giant galaxies (upper curves), and galaxies in the absolute magnitude range from $M_{g}=-20.2$ and $M_{g}=-16.2$ (lower curves).

The sampled $G C$ populations for galaxies brighter than

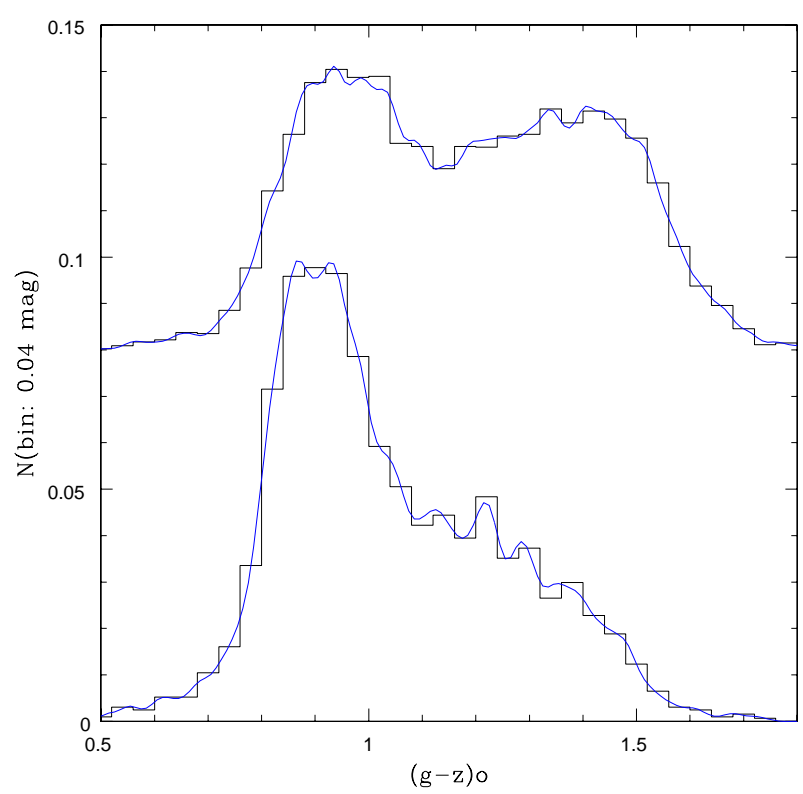

Figure 2. Composite (g-z)o colour distributions for 4506 GCs brighter than $M_{g}=-6.5$ in 10 giant galaxies (upper histogram; shifted upwards arbitrarily) and 3165 GCs in 68 galaxies (fainter than $M_{g}=-20.2$; lower histogram) in the Virgo cluster. The histograms have 0.04 mag bins. The solid curves are smoothed colour distributions after convolving with a 0.015 mag Gaussian kernel. A broad bimodality is seen for GCs in giant galaxies and becomes less evident in fainter galaxies.

$M_{g}=-16.2$ amounts to 7671 GCs in 88 Virgo galaxies and 4317 clusters in 42 Fornax galaxies. In these populations, 4506 clusters belong to ten giant galaxies (i.e. above the $M_{g}=-20.2$ line in Fig. 1) in Virgo and 1789 clusters in five Fornax galaxies.

Both Fig. 2 and Fig. 3 show a result already found and emphasized by Peng et al. (2006): GC colour bimodality becomes more evident as the galaxy brightness increases. These authors, and Jordán et al. (2009), present a description of their method to segregate GCs from field interlopers using the $z$ magnitude $v s$. cluster size diagram (see their Fig. 1).

At our adopted limiting magnitude $(g=25.0)$, and considering the expected colour range for GCs, the limiting $z$ magnitude results close to 24.50. Figure 1 in Peng et al. (2006) indicates that, above this magnitude, the presence of field interlopers is practically negligible (also see Section 8).

\section{COLOUR PATTERN RECOGNITION}

The recognition of eventual patterns arising in feedback phenomena on the GCSs faces a starting problem: The a priori unknown characteristics of these patterns. In a naive way, one might expect that quenching or enhancement of $G C s$ formation would be reflected, respectively, as valleys and peaks on top of the overall cluster formation history, encoded in the $G C C D$, of a given galaxy. Here we adopt a simple strategy based on the identification of peaks and valleys in the composite GCCDs.

Both the Virgo and Fornax galaxies were sampled with a 


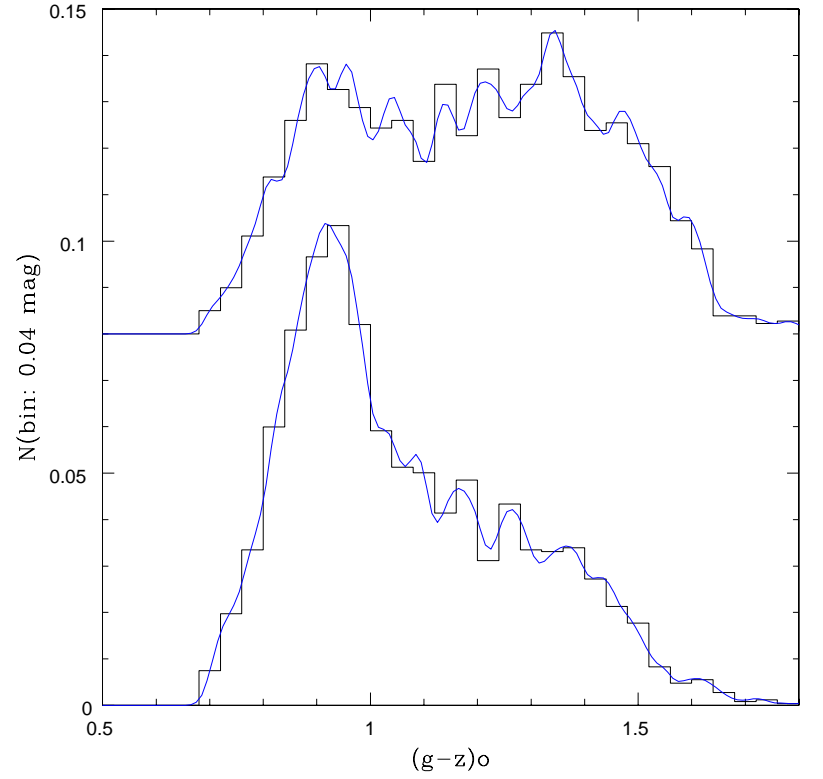

Figure 3. Composite (g-z)o colour distributions for 1789 GCs brighter than $M_{g}=-6.5$ in 5 giant galaxies (upper histogram; arbitrarily shifted upwards) and 2528 GCs in 37 galaxies (fainter than $M_{g}=-20.2$; (lower histogram) in the Fornax cluster. The histograms have 0.04 mag bins. The solid curves are smoothed colour distributions after convolving with a 0.015 mag Gaussian kernel. A broad bimodality is seen for GCs in giant galaxies and becomes less evident in fainter galaxies.

moving window, characterized by a range in galaxy absolute magnitudes $\Delta M_{g}$. This windows moves in steps $\delta_{M_{g}}$. Discrete and smoothed colour distributions were then obtained by convolving the $(g-z) o$ colours of all GCs associated with galaxies in the sampling window with a Gaussian kernel $\sigma_{(g-z)}$. Peaks and valleys in the composite (smoothed) $G C C D s$ where identified at colours were the first derivative $d N / d(g-z) o$ is null.

After exploring the effects of changing the $\left.\Delta M_{g}, \sigma_{(g-z)}\right)$ and $\delta_{M_{g}}$ values, in what follows we adopt values of 0.40 , 0.015 and 0.20 , respectively, for these parameters.

The colour peaks detected within each sampling window are shown, as a function of the mean composite $M_{g}$ magnitude of the galaxies, in Fig. 4 and Fig. 5 for the Virgo and Fornax GCs respectively.

As a first step we analyse galaxies in the magnitude range from $M_{g}=-20.2$ to -16.2 (i.e., non-giant galaxies). The number vs. colour statistics for these peaks in Virgo (237 peaks; upper curve), and Fornax (179 peaks; lower curve), are shown in Fig. 6 in a smoothed format, normalized by the total number of peaks, and adopting the same Gaussian kernel $\sigma_{(g-z)}$, used to derive the GCCDs within the galaxy sampling windows.

A simple cross correlation of these patterns with the composite $G C C D s$ defined by galaxies within a given $\Delta M_{g}$ window, helps in identifying which galaxy groups are the most important contributors to the presence of these patterns.

It is worth mentioning that the colour patterns and the $G C C D s$ are two different types of statistics. The first re-
Table 1. Template (g-z) colour patterns in Virgo and Fornax.

\begin{tabular}{ccc}
\hline \hline Id. Number & (g-z)o Virgo & (g-z)o Fornax \\
\hline 1 & 0.74 & 0.73 \\
2 & 0.85 & 0.94 \\
3 & 0.95 & 1.09 \\
4 & 1.05 & 1.18 \\
5 & 1.13 & 1.25 \\
6 & 1.21 & 1.37 \\
7 & 1.29 & 1.43 \\
8 & 1.39 & 1.50 \\
9 & 1.48 & 1.61 \\
10 & 1.60 & 1.73 \\
11 & 1.72 & - \\
\hline
\end{tabular}

flects the frequency of a given $(g-z)$ colour peak for galaxy groups within a determined range in absolute magnitude. The second, indicates the frequency of $G C s$ with the same colour.

This procedure indicates that the highest correlation signals are obtained, both in Virgo and Fornax, for GCs in galaxies within the five brightest sampling windows, i.e., $M_{g}$ from -20.2 to -18.8 .

Within those windows, the routine finds a total of 80 colour peaks in Virgo and 64 in Fornax. In what follows we refer to the smoothed colour distribution of these peaks as the template Virgo and Fornax patterns (TVP and TFP) respectively.

The template colour patterns are listed in Table 1. From here, the references to a particular colour peak is given within brackets an identified by their number (first column) in this table. Dubious cases are indicated by a colon following the identification number. The TVP and TFP derived from galaxy groups between $M_{g}=-20.2$ to -18.8 , are compared with those corresponding to GCs in galaxy groups fainter than $M_{g}=-18.8$ (159 peaks in Virgo and 115 peaks in Fornax) in Fig. 7 and Fig. 8.

For Virgo, a comparison of both colour patterns shows coincidences in the colour domain of the intermediate-red GCs: peaks $[6,7,8,9,10]$, are comparable to within \pm 0.01 mags in $(g-z)$. Bluer peaks, in turn, do not show a clear correspondence.

The Fornax patterns also show coincidences $[4,5,6,7,8]$, to within $\pm 0.025 \mathrm{mag}$, in the colour range of the intermediate-red GCs. The weaker coincidences, compared to those in Virgo, are possibly a consequence of the smaller number statistics in Fornax. Again, as in the case of the Virgo patterns, the coincidences are not detectable for peaks bluer than $(g-z)=\approx 1.10$. The discrete and smoothed GCCDs for clusters brighter than $M_{g} \approx-6.4$ ( $g=25$ at the distance of the Fornax cluster) in galaxies within the absolute magnitude range $M_{g}$ from -20.2 to -19.2 , are displayed in Fig. 9 (13 Virgo galaxies; 1349 GCs) and in Fig. 10 (7 Fornax galaxies, 1266 GCs). These figures also show the expected statistical count uncertainties for a population level of $\approx 65 G C$ per bin (shown as bar crossed dots).

$G C s$ in these Virgo galaxies exhibit a double blue peak [i.e., clusters bluer than $(g-z) o=1.0$ ] and five colour peaks in the domain of the intermediate-red GCs. In turn, the $G C C D$ in Fornax has a single blue peak and four in the 


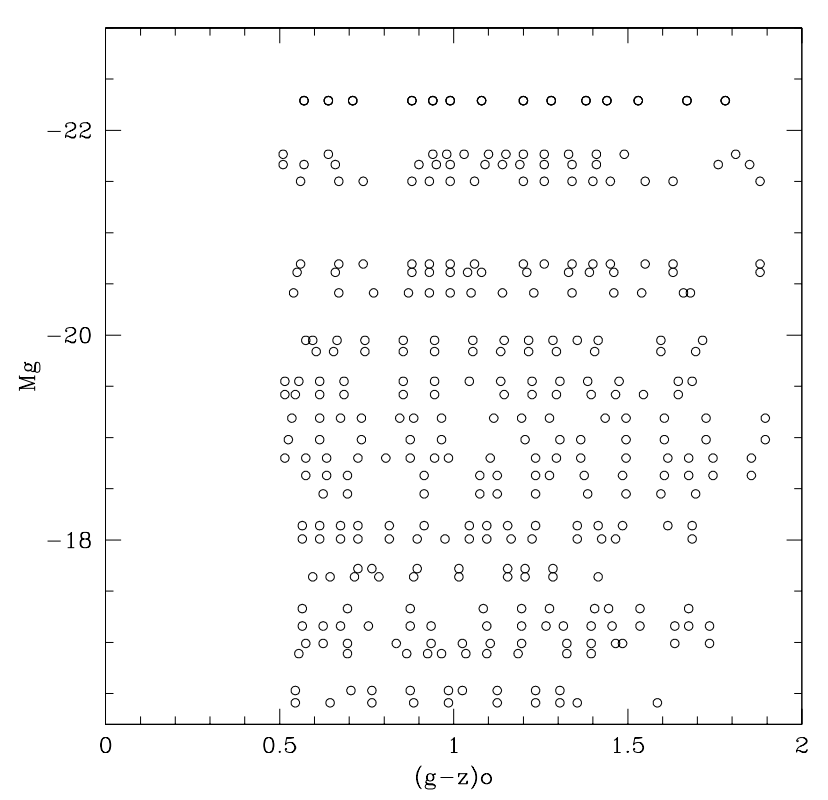

Figure 4. Colour peaks (open circles), detected on composite $G C$ samples for Virgo galaxies, grouped in $0.4 \mathrm{mag}$ bins in $M_{g}$. These colours are shown as a function of the mean composite $M_{g}$ of the galaxies each group.

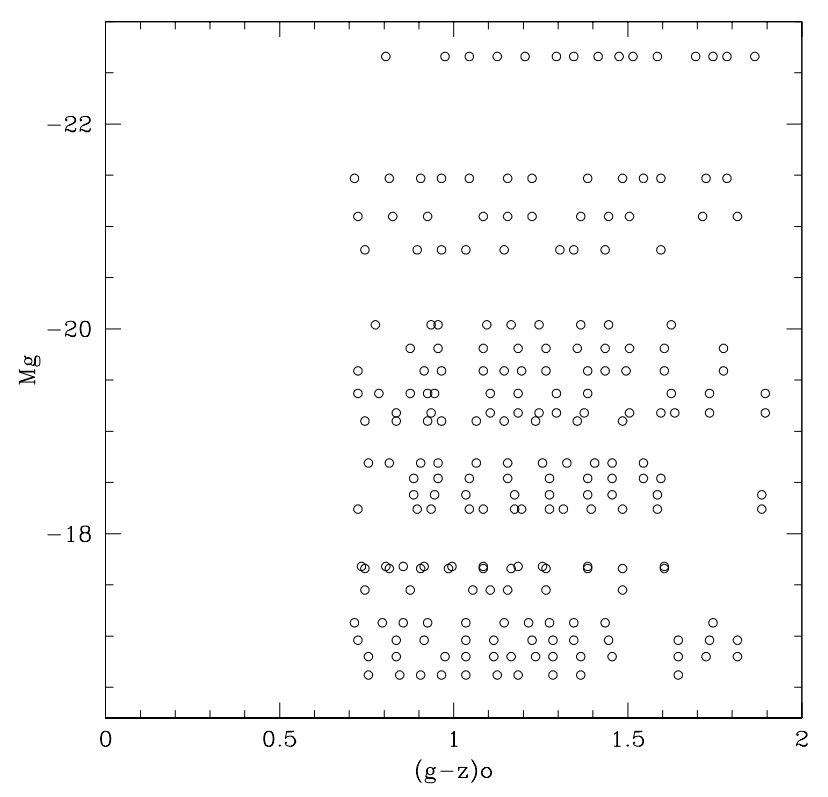

Figure 5. Colour peaks (open circles), detected on composite $G C$ samples for Fornax galaxies, grouped in 0.4 mag bins in $M_{g}$. These colours are shown as a function of the mean composite magnitude $M_{g}$ of the galaxies in each group.

intermediate-red GCs colour range. In both cases, the amplitude of the fluctuations are comparable to the expected statistical uncertainties.

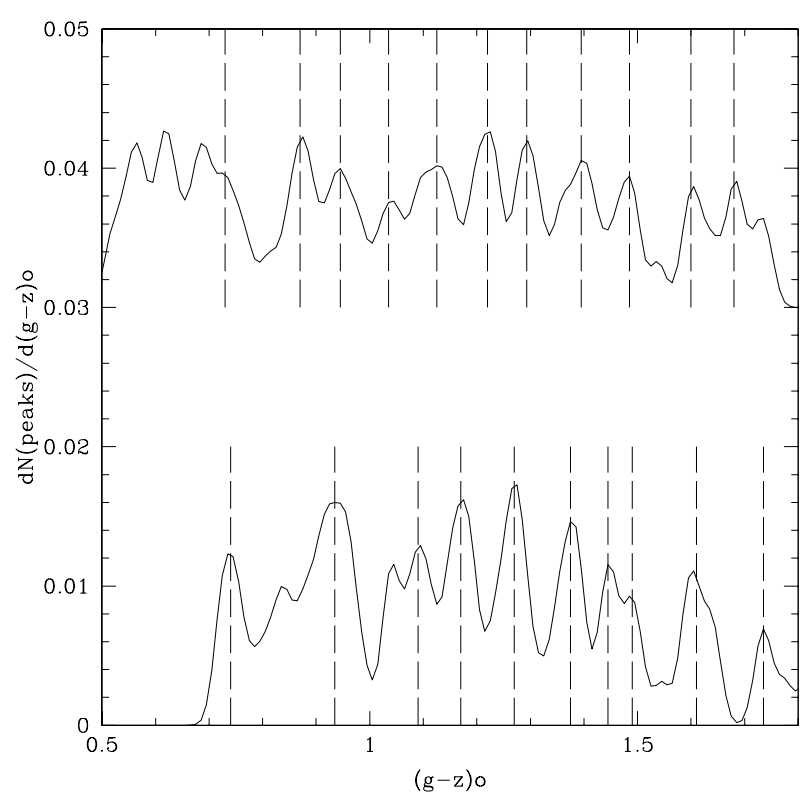

Figure 6. Smoothed distributions of the colour peaks (shown in Fig. 4 and Fig. 5) after a convolution with a 0.015 mag Gaussian kernel. The upper curve corresponds to GCs in non-giant Virgo galaxies (arbitrarily shifted upwards) and the lower one to GCs in non-giant Fornax galaxies. Dashed lines identify the colour of each peak in the different patterns.

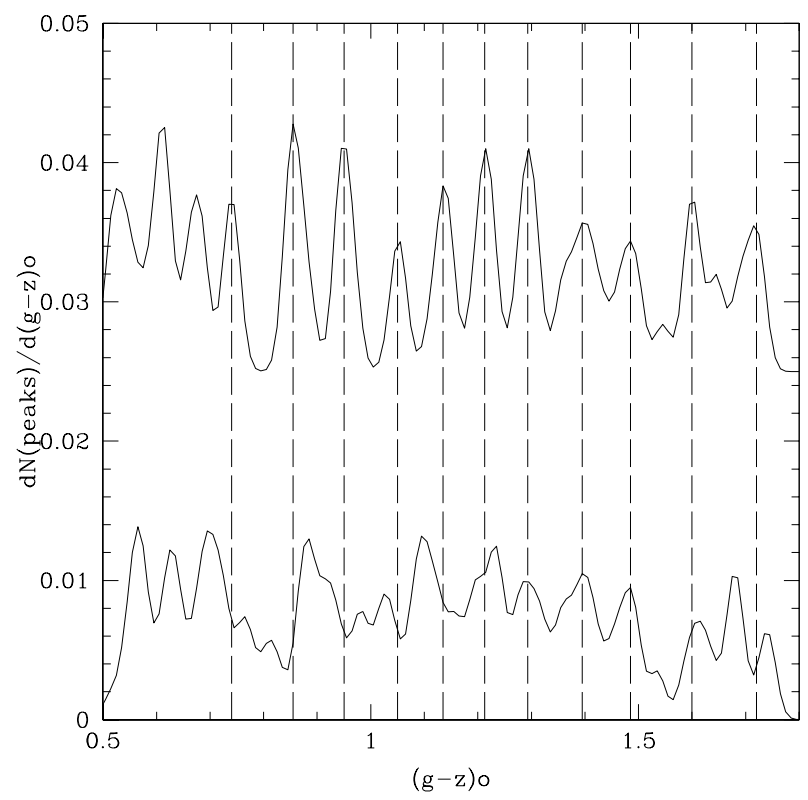

Figure 7. Smoothed colour distribution for 80 colour peaks detected in $G C s$ associated with non-giant Virgo galaxies brighter than $M_{g}=-18.8$ (upper curve; shifted arbitrarily upwards) compared with those corresponding to 141 peaks found in fainter galaxies (lower curve). The dashed lines indicate the colours corresponding to peaks in the the upper curve. 


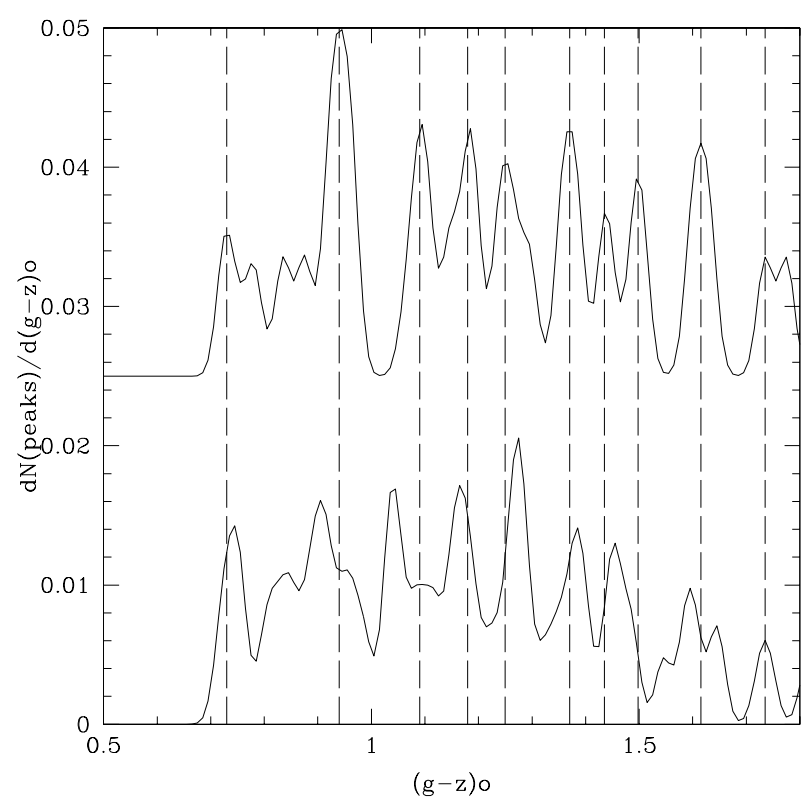

Figure 8. Smoothed colour distribution for 64 colour peaks detected in $G C s$ associated with non-giant Fornax galaxies brighter than $M_{g}=-18.8$ (upper curve; arbitrarily shifted upwards) compared with those corresponding to 115 peaks found in all the fainter galaxies (lower curve). The dashed lines indicate the peak colours corresponding to the upper curve.

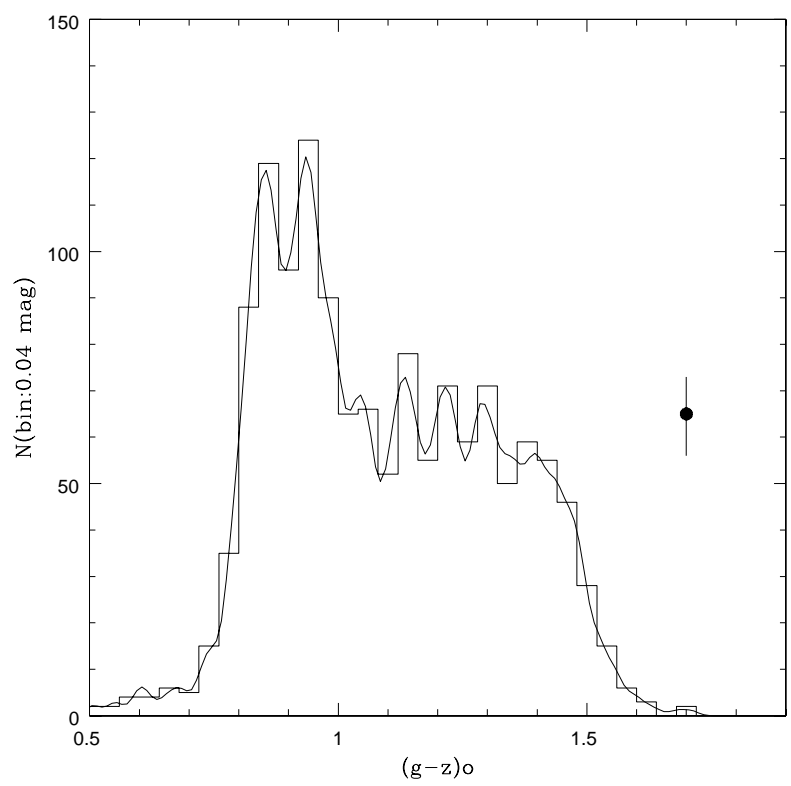

Figure 9. Discrete and smoothed (g-z)o colour distribution for clusters brighter than $M_{g} \approx-6.4$. The sample includes 1349 GCs in 13 Virgo galaxies with $M_{g}$ between -20.2 and -19.2. The histogram has $0.04 \mathrm{mag}$ bins while the smoothed distribution corresponds to a $0.015 \mathrm{mag}$ Gaussian kernel. The filled circle at right corresponds to a $G C$ level of 65 clusters per bin. The vertical line indicates the expected counting uncertainty.

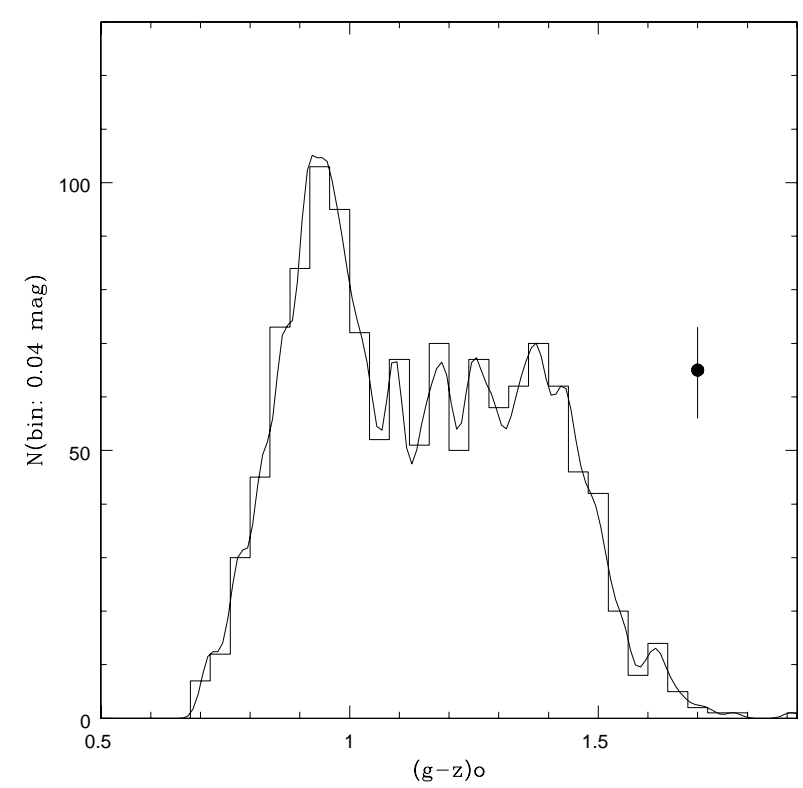

Figure 10. Discrete and smoothed (g-z)o colour distribution for clusters brighter than $M_{g} \approx-6.4$. the sample includes $1266 \mathrm{GCs}$ in 7 Fornax galaxies with $M_{g}$ between -20.2 and -19.2. The histogram has $0.04 \mathrm{mag}$ bins, while the smoothed distribution corresponds to a 0.015 mag Gaussian kernel. The filled circle at right corresponds to a $G C$ level of 65 clusters per bin. The vertical line indicates the expected counting uncertainty.

\section{GC COLOUR DISTRIBUTIONS IN NON-GIANT GALAXIES.}

In this section we analyse the behaviour of the GCCDs in galaxies fainter than $M_{g}=-20.2$, and leave the analysis of some relevant giant galaxies for a following Section. All the $G C C D s$ presented in what follows are normalized by the total number of clusters in each sample.

\subsection{GCs in Virgo Galaxies.}

\subsubsection{GCCD for galaxies with $M_{g}=-20.2$ to -19.2}

The composite $G C C D$ corresponding to thirteen galaxies includes 1513 GCs brighter than $g=25.0$ and is displayed in Fig. 11. Dashed lines in this, and in the following diagrams of this subsection, correspond to the $T V P$. Filled dots indicate the colour peaks, found by the routine described in Section 4. The routine also computes the difference between the colour of each peak and the nearest colour peak in the corresponding template pattern. An overall $\mathrm{rms}$ of the differences is obtained taking into account all the detected peaks. Then, a small colour shift is allowed (for the whole set of detected peaks) in order to minimize that $r m s$. This shift may be justified, for example, in terms of the uncertainties in interstellar reddening. Alternatively, the shift may reflect a difference in chemical abundance between $G C s$ in a given galaxy and those in galaxies that define the template patterns.

In the case of the $G C$ sample shown in Fig. 11, the TVP shows six coincidences, $[2,3,4,5,6,7]$, to within 0.01 mag., with the peaks shown by the $G C C D$, and without 


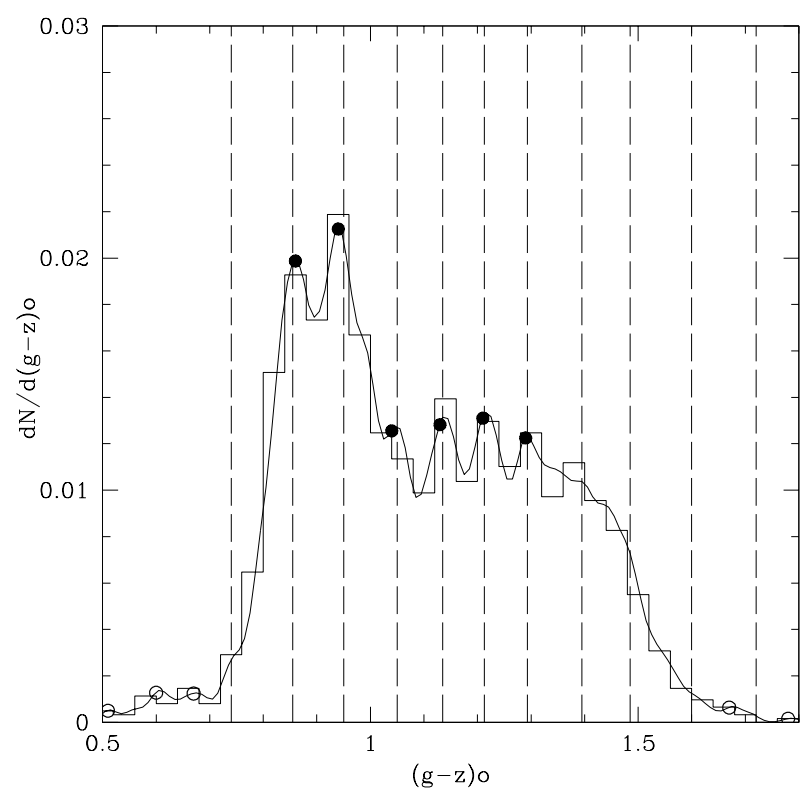

Figure 11. Smoothed (g-z)o colour distribution (adopting a 0.015 mag Gaussian kernel) corresponding to 1513 GCs in 13 Virgo galaxies with $M_{g}-20.2$ to -19.2 compared with the TVP colours (dashed lines; see text).

requiring a colour shift. This result can be expected as most of these galaxies are included in the galaxy groups that define the $T V P$. In turn, splitting the galaxy group in two sub-groups (brighter and fainter than $M_{g}=-19.7$ ) leads to the GCCDs shown in Fig. 12 .

The brighter galaxy group includes six galaxies (VCC 759, 1030, 1062, 1154, 1692, 2092; upper histogram) and 689 GCs while, the fainter one, has seven galaxies (VCC 369, 944, 1279, 1664, 1720, 1938, 2000; lower histogram) and an almost identical number of 682 GCs.

Both GCCDs exhibit coincidences with the TVP. Peaks $[1,2,3,4,5,6,7]$ are detected in the brighter galaxy groups while peaks $[2,3,4,5,6,7,8,9]$ appear on the fainter group. In all these cases the agreement with the TVP colours is better than $\pm 0.01 \mathrm{mag}$. (and zero colour shift).

None of these galaxies, except VCC 1664 (NGC 4564), show a total coincidence of their $G C C D$ with the $T V P$ when analysed as individuals. In the case of VCC 1664, that contributes with 8 percent of the total $G C$ sample, there are eight coincidences $[2,3,4,5,6,7,8,9]$, with colour differences within \pm 0.015 mag. The larger colour differences in this galaxy are possibly an statistical effect resulting from the smaller size of the $G C$ sample.

The analysis of the whole 1513 GCs sample can be also performed in terms of the $g$ apparent magnitude. For objects brighter than $g=23.0$ the $G C C D$ is dominated by the double blue peak while redder GCs (see Fig. A1) exhibit a rather poor coincidence with the $T V P$.

In turn, splitting the $G C$ sample corresponding to objects fainter than $g=23.0$ in two equal number samples: 458 clusters with $g=23$ to 23.75 and 450 clusters with $g=23.75$ to 24.50 (see Fig. A2) allows the identification of the TVP peaks $[2,3,5,6,7]$ and $[2,3,4,5,6,7,8,9,10]$ respectively.

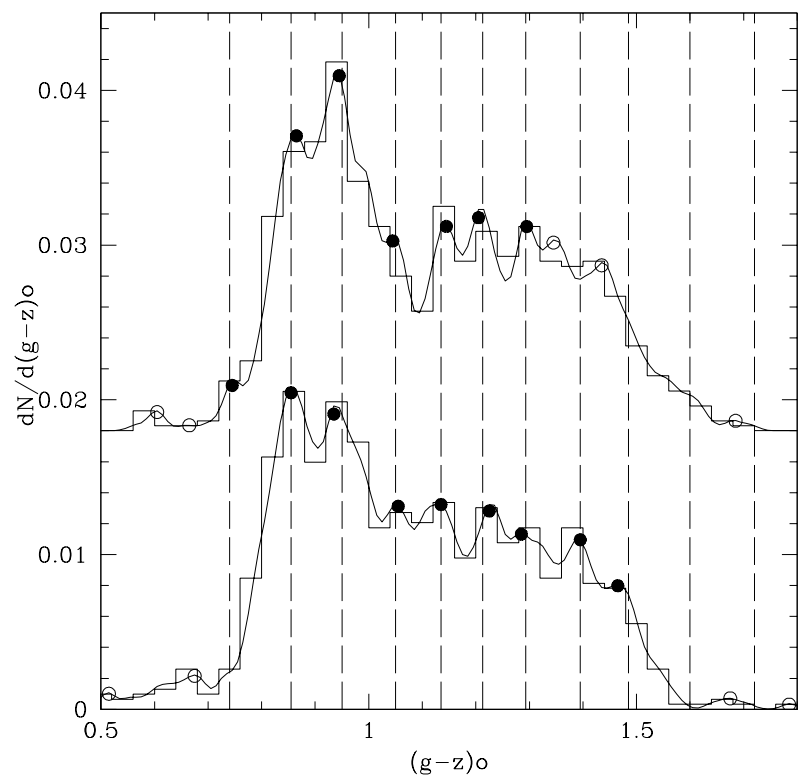

Figure 12. Smoothed (g-z)o colour distribution (adopting a 0.015 mag Gaussian kernel) corresponding to 773 GCs in 6 Virgo galaxies with $M_{g}-20.2$ to -19.7 (upper histogram; shifted arbitrarily upwards) and 762 GCs in 7 galaxies with $M_{g}=-19.7$ to -19.2 (lower curve). The dashed lines corresponds to the TVP colours.

\subsubsection{GCCD for galaxies with $M_{g}=-19.2$ to -18.2}

This group includes 883 GCs in 17 galaxies an the corresponding $G C C D$ is displayed in Fig. 13. This distribution shows a broad blue peak (two overlapped components?) and three approximate coincidences $[6,7,8:]$ with the $T V P$.

\subsubsection{GCCD for galaxies with $M_{g}=-18.2$ to -17.2}

The GCCD is depicted Fig. 14 and corresponds to 673 GCs in 16 galaxies. The blue peak becomes narrower and better defined than in the previous group and no further coincidences with the TVP are detectable. This galaxy sample includes VCC 1297 ( $N G C 4486 B$ ), an object with a relatively large number of $G C s$ for its brightness. Possibly, its cluster population includes a fraction of $G C s$ that in fact belong to the nearby $N G C$ 4486. Removing VCC 1297 from the sampling window, however, does not change the shape of the composite $G C C D$ in a significant way.

\subsubsection{GCCD for galaxies with $M_{g}=-17.2$ to -16.2}

Fig. 15 depicts the GCCD corresponding to 453 GCs in 22 galaxies. This $G C s$ sample exhibits a single blue peak, similar to that seen in the previous group. Three other TVP peaks $[5,6,7]$ seem to be marginally present.

\subsection{GCs in Fornax galaxies}

In this subsection, we repeat a similar analysis for $G C s$ in Fornax galaxies. 


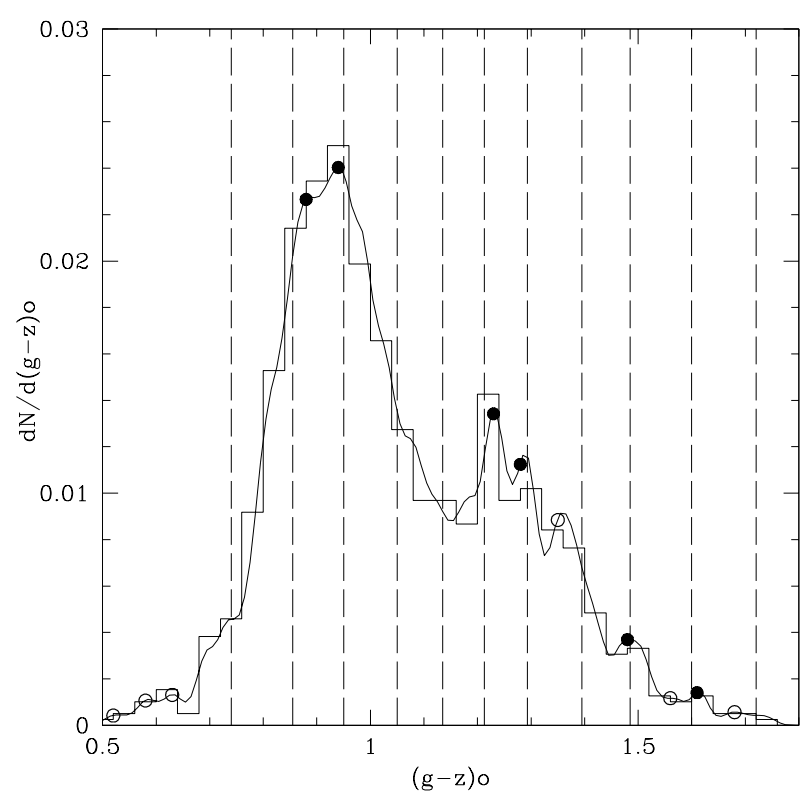

Figure 13. Smoothed (g-z)o colour distribution (adopting a 0.015 mag Gaussian kernel) corresponding to 883 GCs in 17 Virgo galaxies with $M_{g}-19.2$ to -18.2 compared with the colours of the TVP (dashed lines; see text).

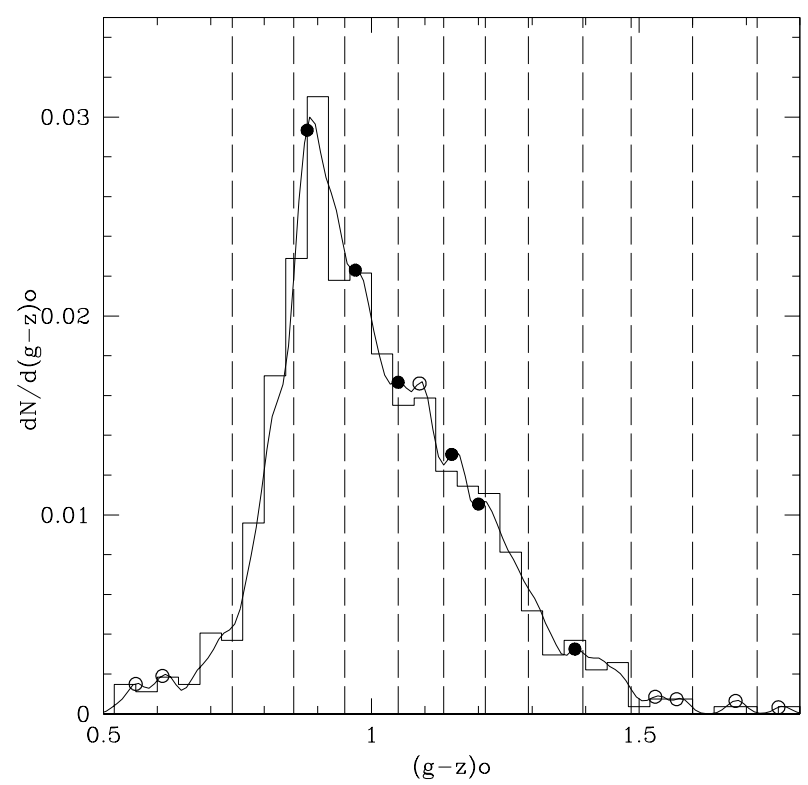

Figure 14. Smoothed (g-z)o colour distribution (adopting a 0.015 mag Gaussian kernel) corresponding to 673 GCs in sixteen Virgo galaxies with $M_{g}-18.2$ to -17.2 compared with the TVP colours (dashed lines; see text).

\subsubsection{GCCD for galaxies with $M_{g}=-20.2$ to -19.2}

The GCCD is displayed in Fig. 16 and includes 1270 GCs in 7 galaxies. This distribution shows seven coincidences with the TFP, $[2,3,4,5,6,7,9]$ within \pm 0.01 or less. As in the case

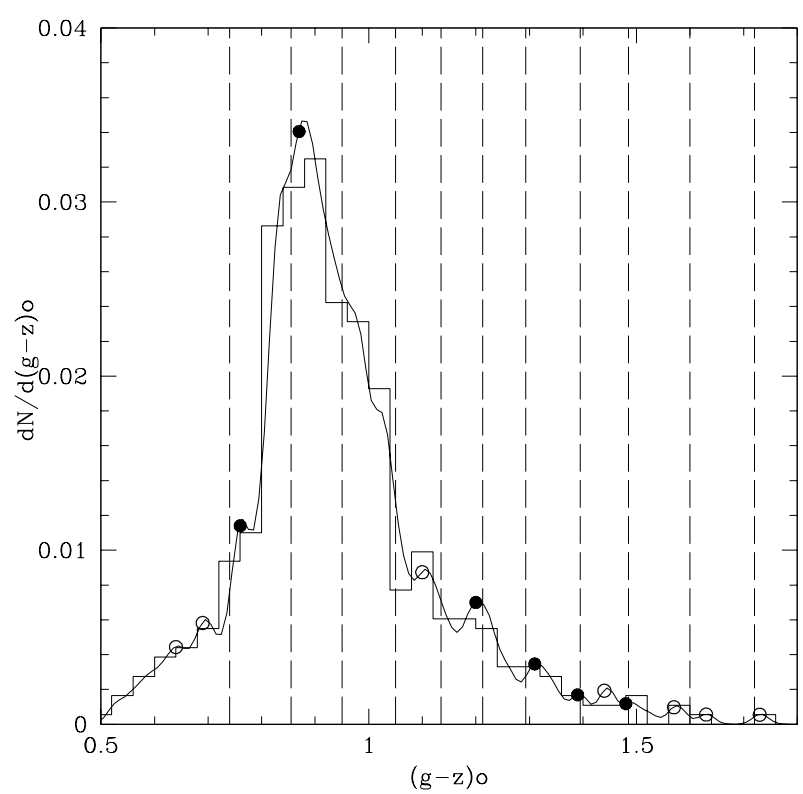

Figure 15. Smoothed (g-z)o colour distribution (adopting a 0.015 mag Gaussian kernel) corresponding to 453 GCs in 22 Virgo galaxies with $M_{g}-17.2$ to -16.2 (upper histogram) compared with the TVP colours (lower curve; see text).

of the Virgo galaxies, this agreement can be expected as most of these galaxies define the TFP.

In turn, the $G C C D$ for 594 clusters in galaxies in the range $M_{g}=-20.2$ to -19.7 (FCC 147, 276, 2006) and for 676 $G C s$ in four galaxies in the range $M_{g}=-20$ to -19.2 (FCC $63,83,184,193)$ is displayed in Fig. 17. In this case the colour distributions indicate eight $[2,3,4,5,6,7,8,9]$ and seven $[1,2,3,4,5,6,9]$ coincidences within \pm 0.01 mags., respectively.

A comparison of the $G C C D s$ for clusters brighter and fainter than $g=23$ in the whole sample (1270 clusters; see Fig. A3) shows that the colour pattern is better defined for the bright clusters (in contrast with the results from the Virgo galaxies that have an opposite behaviour).

The GCCDs for two sub samples with equal number of clusters (see Fig. A4) show that, for the fainter clusters, the blue peak is broader and more poorly defined than its brighter clusters counterparts. For redder $G C s$, the patterns display five coincidences in common: $[3,4,5,6,7]$. As in the case of Virgo, the TFP can be recognized when splitting the $G C$ samples in terms of galaxy groups or in terms of apparent $g$ magnitude.

\subsubsection{GCCD for galaxies with $M_{g}=-19.2$ to -18.2}

Fig. 18 shows the $G C C D$ of 856 GCs in 17 galaxies. The blue peak appears located at the expected position of the TFP. Three mildly modulated features are seen towards the red but they are not clearly coincident with the $T V P$.

\subsubsection{GCCD for galaxies with $M_{g}=-18.2$ to -17.2}

Fig. 19 corresponds to 151 GCs in six galaxies (FCC 55, 95, 143, 152, 301, 335). Besides the prominent blue peak, there are other three well defined peaks at the expected position in 


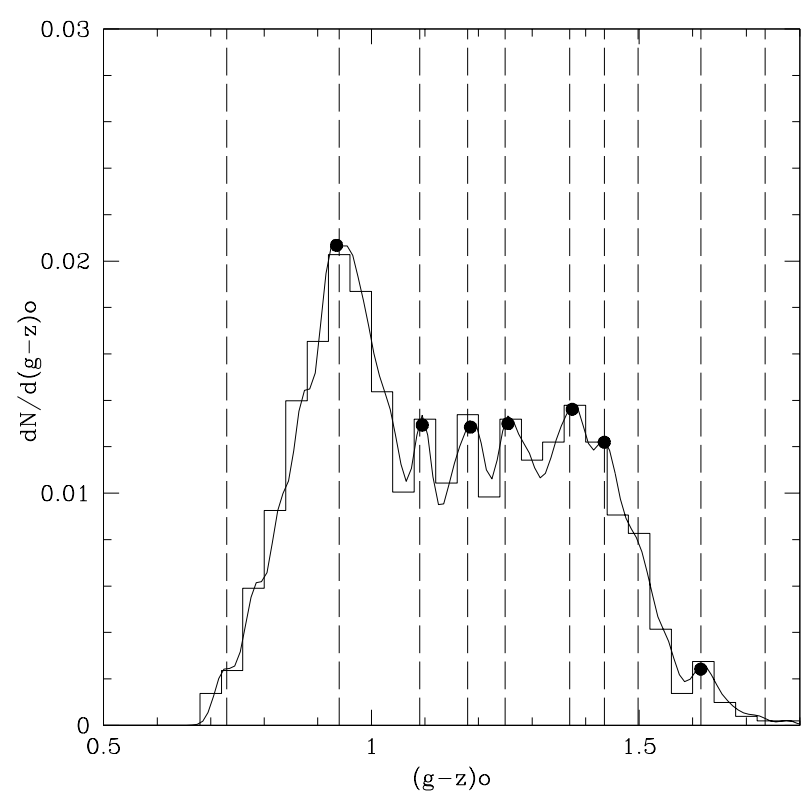

Figure 16. Smoothed (g-z)o colour distribution (adopting a 0.015 mag Gaussian kernel) corresponding to 1270 GCs in seven Fornax galaxies with $M_{g}-20.2$ to -19.2 compared with the colours of the TFP (dashed lines; see text).

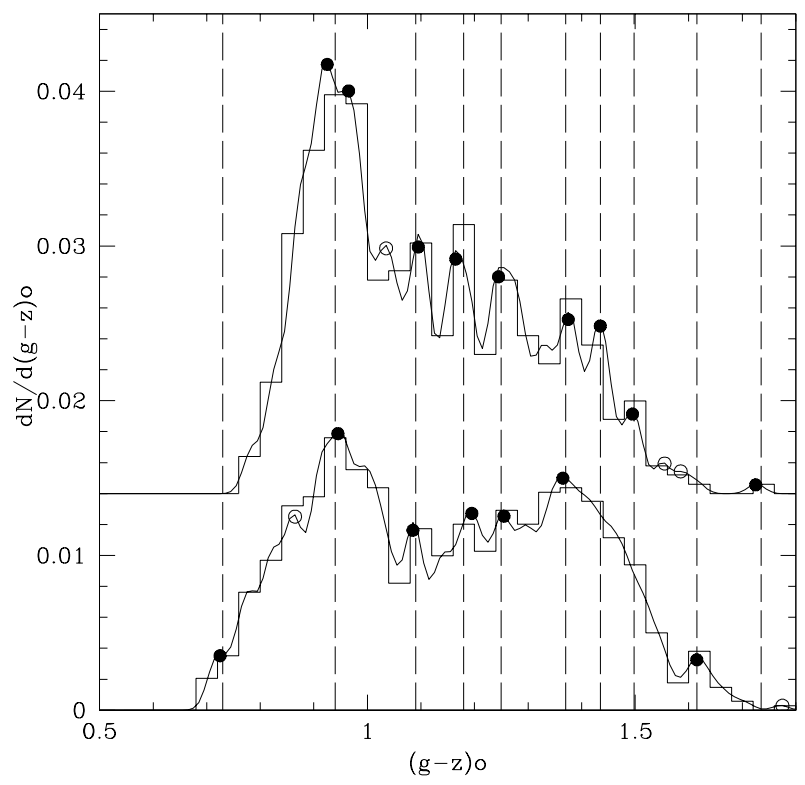

Figure 17. Smoothed (g-z)o colour distribution (adopting a 0.015 mag Gaussian kernel) corresponding to 594 GCs in 3 Fornax galaxies with $M_{g}-20.2$ to -19.7 (upper curve, arbitrarily shifted upwards) and 676 GCs in 4 galaxies with $M_{g}-19.7$ to -19.2 (lower curve). The dashed lines corresponds to the TFP colours (dashed lines).

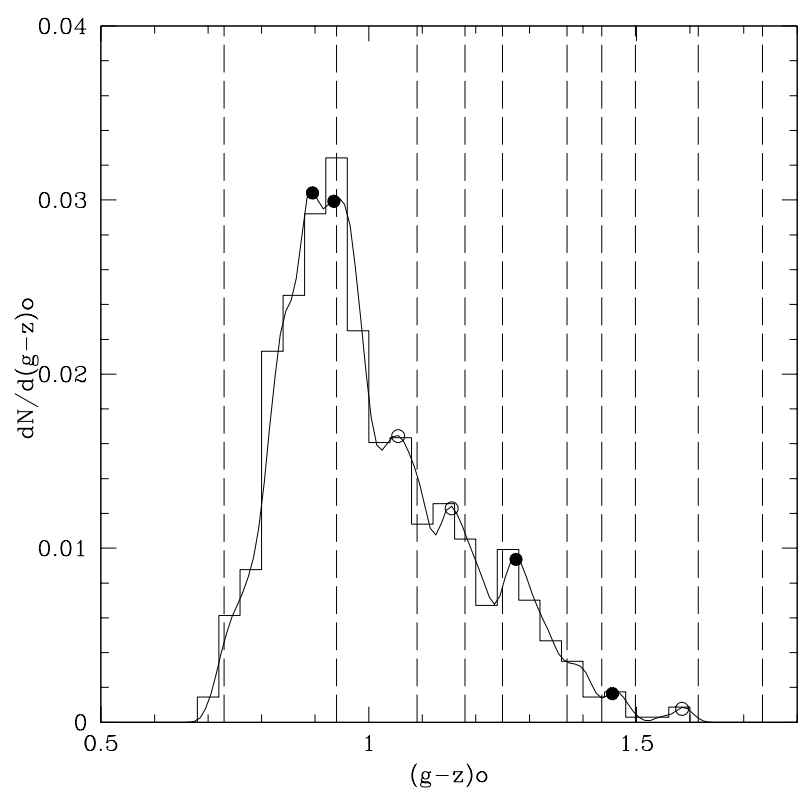

Figure 18. Smoothed (g-z)o colour distribution (adopting a 0.015 mag Gaussian kernel) corresponding to 856 GCs in 17 Fornax galaxies with $M_{g}-19.2$ to -18.2 compared with the colours of the TFP (dashed lines; see text).

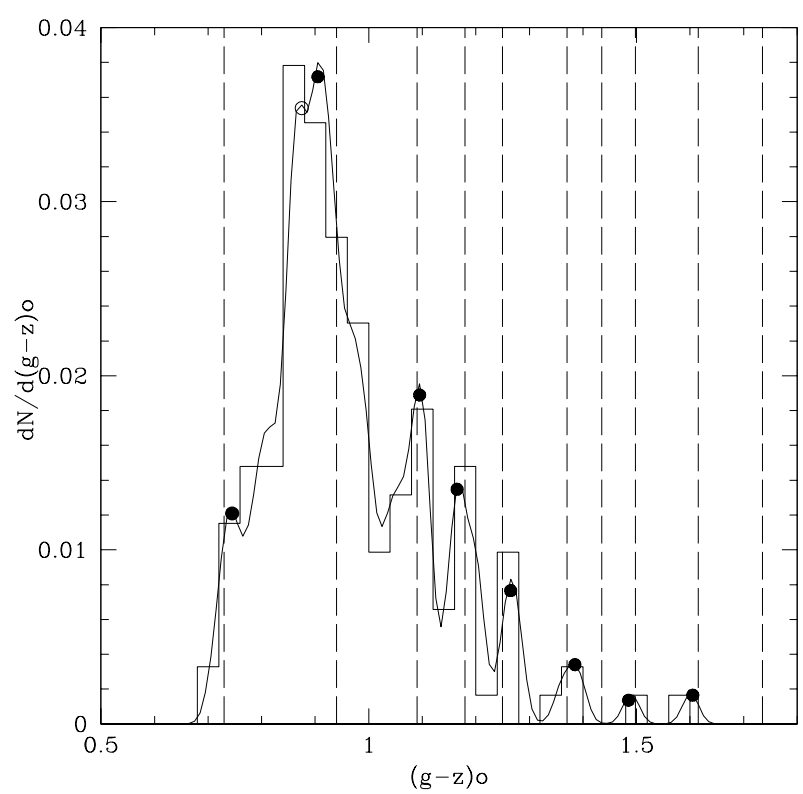

Figure 19. Smoothed (g-z)o colour distribution (adopting a 0.015 mag Gaussian kernel) corresponding to 152 GCs in six Fornax galaxies with $M_{g}-18.2$ to -17.2 compared with the colours of the TFP (dashed lines; see text).

the TFP $[3,4,5]$ with colour differences within \pm 0.01 mag. We stress that these galaxies are not included in the sample that defines the TFP (which are about two magnitudes brighter). Remarkably, the four peaked pattern seen in this group survives when the sample is split in terms of apparent magnitude or galactocentric radius. 


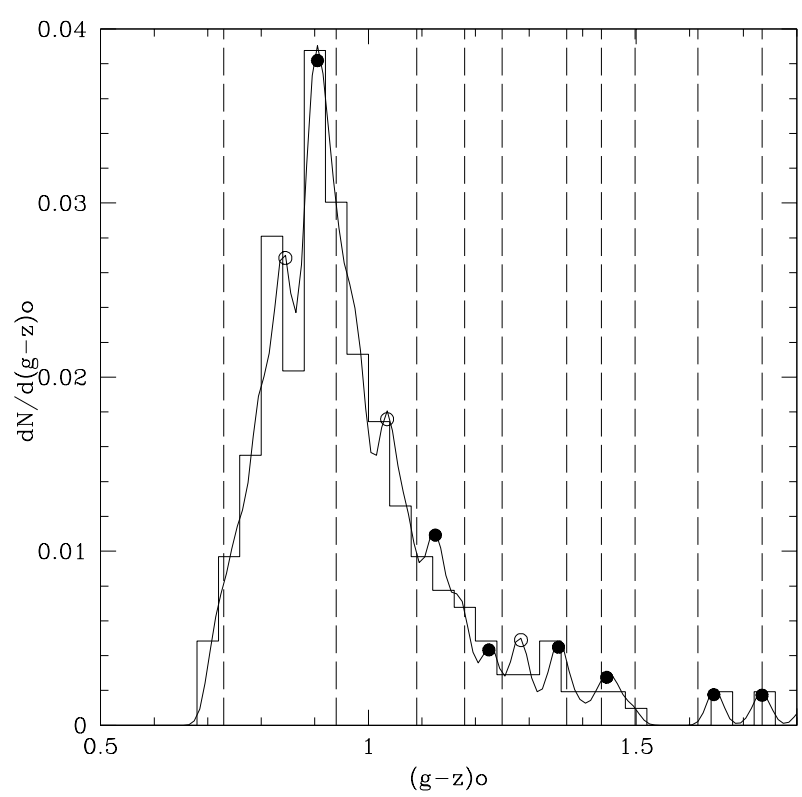

Figure 20. Smoothed (g-z)o colour distribution (adopting a 0.015 mag Gaussian kernel) corresponding to 258 GCs in 13 Fornax galaxies with $M_{g}-17.2$ to -16.2 compared with the colours of the TFP (dashed lines; see text).

\subsubsection{GCCD for galaxies with $M_{g}=-17.2$ to -16.2}

The GCCD is shown in Fig. 20 and includes 258 GCs in 13 galaxies. This distribution is very similar to that corresponding to the previous galaxy group, except for the lack of any evident feature that could be compared with those in the TVP (except the blue peak).

\section{THE CENTRAL GALAXIES IN VIRGO AND FORNAX}

In this section we discuss the results for $N G C 4486$ and $N G C 1399$ given their condition of central galaxies in Virgo and Fornax, respectively, as well as some preliminary results about other bright galaxies included in the Appendix.

For these galaxies we performed an analysis similar to that described in Section 4. In this case, the routine produces $G C C D s$ within given ranges of galactocentric radii and position angles, and looks for similarities with the corresponding template patterns.

A similar analysis was performed on an independent photometric data set of $G C$ candidates both in NGC 4486 and NGC 1399 presented in Forte et al. (2007).

Adopting a limiting magnitude $T_{1}=23.6$ (that corresponds to $g \approx 24.5)$ produces colour errors comparable to those of the $A C S$ photometry at $g=25.0$. The transformation of $(C-$ $\left.T_{1}\right)$ to $(g-z)$ colours was derived from a peripheral field in $N G C$ 4486, that includes some $500 G C$ candidates with both Washington and griz' photometry (Forte et al. 2013). A revision of the data in this last paper leads to a bi-sector fit:

$$
(g-z)=0.755\left(C-T_{1}\right)-0.058
$$

(with slope and zero point errors of \pm 0.073 and \pm 0.012 , respectively).

Taking into account the slope of this colour-colour relation, we adopt a Gaussian kernel of 0.02 mag (instead of 0.015 mag) to obtain the smoothed $\left(C-T_{1}\right) G C C D$ and then transform this colour to $(g-z)$ through the last equation.

\subsection{Globular cluster colour distribution in NGC 4486.}

Fig. 21 displays the $G C C D$ for clusters in $N G C 4486$ both in the discrete and smoothed $\left(\sigma_{(g-z)}=0.015\right)$ histogram formats. These GCs (1395 objects) are brighter than $g=25.0$ and within an annular region with galactocentric radii from 0 to 120 ". This figure shows a broad bimodal structure with mild modulations that peak close to some of the colours that are defined in the $T V P$.

Within this sample, the peak finding routine indicates that the best match to the $T V P$ occurs for $G C s$ in a galactocentric range from 40 to 120 " and position angles (North to East) $P A$ between 30 and $150^{\circ}$. The GCCD for 414 clusters in this region are shown in Fig. 22. There are nine colour coincidences $[2,3,4,5,6,7,8,9,10]$ with the $T V P$ to within \pm $0.01 \mathrm{mag}$ and without requiring any colour shift.

The same analysis was carried out using the $\left(C-T_{1}\right) o$ colours presented in Forte et al. (2007). These data, however, have a lower spatial coverage than that of the $A C S$ in the central region of the galaxy (i.e. galactocentric radii smaller than $\approx 40^{\prime \prime}$ ) due to image saturation.

The GCCD displayed in Fig. 23, corresponds to 683 $G C s$, with $\left(C-T_{1}\right) o$ colours from 0.90 to 2.2 , and within an annular region defined by galactocentric radii from 90 to 150 ". The best agreement with the TVP for peaks $[2,3,4,5,6,7,8:]$ corresponds to a colour shift of -0.02 mag.

A further confirmation of the presence of the $T V P$ in GCs associated with NGC 4486 is provided by another independent data set. In this case the $(g-z)^{\prime}$ photometry comes from Gemini - GMOS photometry given in Forte et al. (2013) for clusters brighter than $g \approx 23.4$, and used to establish the $\left(C-T_{1}\right)-(g-z)$ relation presented in this section.

The $G C C D$ corresponding to this field (see Fig A5), shows several peaks coincident with those in the TVP, $[2,3,4:, 5,6,7]$, with colour differences within $\pm 0.01 \mathrm{mag}$. It is worth mentioning that the presence of a multi-peak $G C C D$ in $N G C 4486$ can be seen also in the $U V$ photometry presented by Bellini et al. (2015). In particular, the GCCD corresponding to their $\left(m_{F 275 W}-m_{F 814 W}\right)$ colour index (see Fig. 6 in this last paper) also displays 6 to 8 peaks. Further analysis of this result, in the context of this paper, is currently under way.

Even though the results of other giant galaxies in Virgo, for which extensive photometric data is available in the literature, will be discussed elsewhere (Forte et al. in prep.), some of them are included in the Appendix. For example, the $G C C D$ for the brightest galaxy in Virgo, NGC 4472 exhibits nine colour peaks $[2,3,4,5,6,8,9,10,11]$ within \pm 0.015 mag from those defined by the $T V P$ (after applying a colour shift of -0.05 mag; see Fig A6). Peaks $[1,7]$ are also detectable but exhibit colour differences larger than 0.02 mag. when compared with the nearest peak in the template pattern. This sample includes 517 GCs with galactocentric radii from 20 to $110 "$. 


\subsection{Globular cluster colour distribution in NGC 1399}

The smoothed and discrete $G C C D$ for the giant galaxy $N G C$ 1399, displayed in Fig. 24, corresponds to the $A C S$ photometry of 814 GCs within a galactocentric radius of 120 ", after adopting a colour shift of $+0.01 \mathrm{mag}$.

The coincidences with the TFP are more evident for a sub-sample that includes 309 objects with galactocentric radii from 0 to $90^{\prime \prime}$ and $P A$ from 90 to $270^{\circ}$ (i.e. mainly to the south of the galaxy centre) shown in Fig. 25. The GCCD, after applying a colour shift of +0.02 mag., exhibits eight coincidences $[2,3,4,5,6,7,8,9]$ to within \pm 0.015 mag with the TFP.

In turn, Fig. 26 presents the GCCD derived from $\left(C-T_{1}\right)$ colours, including 293 clusters in a galactocentric range from 0 to $120^{\prime \prime}$ and $P A 90$ to $360^{\circ}$. As in the previous diagram, several coincidences with the TFP can be detected to within \pm 0.015 : $[3,4,5,6,7,8,9]$. Peaks $[1,2]$ are also detectable but exhibit colour differences larger than 0.025 mag. when compared with the nearest peak in the template pattern. This $G C s$ sample partially overlaps with that corresponding to the previous diagram but, due to image saturation, is incomplete below a galactocentric radius of $\approx 50$ "I

Other relevant cases, among the bright galaxies in Fornax, are $N G C 1404$, located $\approx 15^{\prime}$ from $N G C 1399$ on the sky, and NGC 1316, the brightest galaxy in the Fornax cluster. This galaxy has a complex $G C S$ including several cluster populations (Goudfrooij et al. 2001; Richtler et al. 2012; Sesto et al. 2016).

The $G C C D$ of the first of these galaxies exhibits nine coincidences $[1,2,3,4,5,6,7,8,10]$ with the TFP (see fig. A7) that show up on top of what seems to be a rather bimodal colour distribution, a situation that is similar to that of the GCCD in NGC 4472 (see Fig. A6). Finally, Fig. A8 shows the $G C C D$ for a sample of $367 G C s$ with galactocentric radii from 40 to 120 " in NGC 1316. Again, and top of a blue ward skewed colour distribution, there are several peaks coincident with the TFP

\section{TENTATIVE MULTI-POPULATION FITS}

Bimodal $G C$ colour distributions are usually represented in terms of Gaussians that fit the blue and red $G C$ populations as shown, for example, in Harris et al. (2017). A different approach links chemical abundance, and $G C$ integrated colours assuming exponential dependences of the number of clusters with $\left(Z / Z_{\odot}\right)$ and colours (e.g. VanDalfsen \& Harris 2004; Forte et al. 2007)

Alternatively, the appearance the composite GCCDs for galaxies with $M_{g}=-20.2$ to -19.2 suggests that these distributions could be matched in an extreme case, just in terms of discrete mono-colour $G C$ populations.

In order to address this issue, we first determined the photometric behaviour of a mono-colour $G C$ family, after adding the photometric errors and the effect of the Gaussian smoothing kernel. In what follows, we identify this function as the "colour spread function" $(C S F)$.

Each colour corresponds, for a given age, to a chemical abundance $[Z / H]$. In this approach we adopt the colourmetallicity relation defined by the $S S P$ models presented by

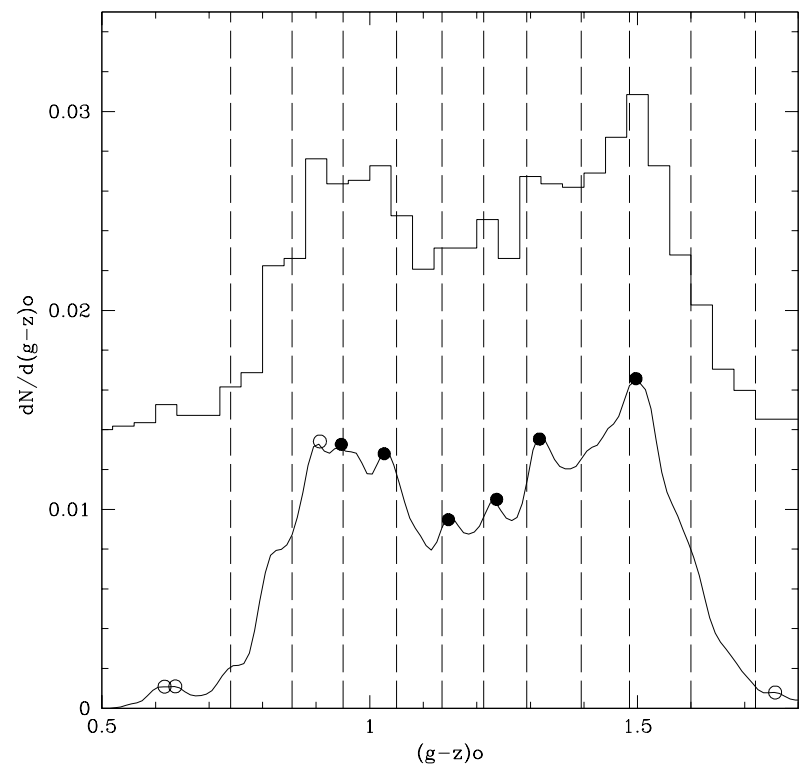

Figure 21. Discrete histogram (arbitrarily shifted upwards) and smoothed colour distribution for $1395 G C$ candidates with galactocentric radii from 0 to $120^{\prime \prime}$ in NGC 4486. Dashed lines correspond to the TVP (see text).

Bressan et al. (2012), for an age of 12 Gy (but see Section 8), and a Salpeter integrated $G C$ luminosity function.

These models, after a small correction to the $g$ magnitudes (see Sesto et al. 2016), give a good match to the $G C$ multi-colour relations observed in the peripheral field of NGC 4486 (Forte et al. (2013).

Photometric errors as a function of the $g$ and $z$ magnitudes were modelled adopting the values given in Table 4 and Table 2 of Jordán et al. (2009) and Jordán et al. (2015) for $G C s$ in Virgo and Fornax galaxies.

For galaxies with $M_{g}=-20.2$ to -19.2 the $G C$ integrated luminosity function can be characterized by a Gaussian with a $\sigma$ parameter of $\approx 1.0$ mag. (Villegas et al. 2010) and turnovers at $g=23.85$ (Virgo) and $g=24.26$ (Fornax).

The procedure starts generating Monte-Carlo apparent $g$ magnitudes, controlled by the Gaussian integrated luminosity function, in a magnitude range from $g=20.0$ to 25.0 and with a given metallicity. Once a model $(g-z)$ colour is obtained from the colour-metallicity relation, a $z$ magnitude is derived. Model errors for both the $g$ and $z$ magnitudes are added, and an "observable" colour is derived from the magnitudes difference. These colours were, in turn, smoothed with a 0.015 mag Gaussian kernel (adopted in the analysis described in previous Sections).

The resulting $C S F$ functions for the Virgo and Fornax datasets are depicted in Fig. 27. for three reference $[Z / H]$ values $(-2.0 ;-0.7 ; 0.0)$.

The fitting routine initially assigns equal amplitudes to all components, and mean colours coincident with those of the observed peaks in the corresponding $G C C D$. After computing the observed minus model difference, each component is changed iteratively allowing for a variation of its initial amplitude and colour. The adopted solution is the one that minimizes the rms of the observed minus model difference. 


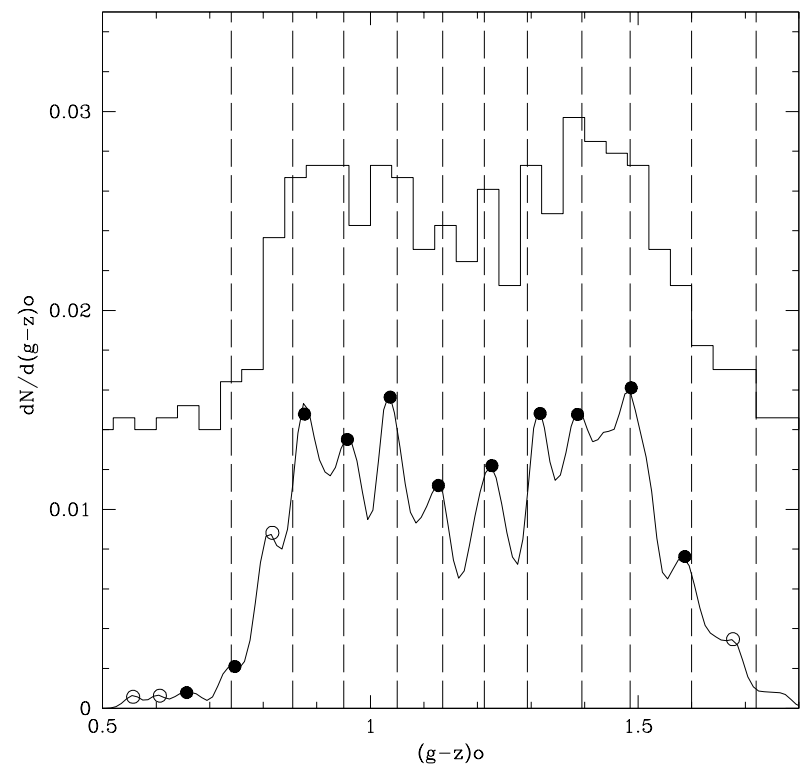

Figure 22. Discrete histogram (arbitrarily shifted upwards) and smoothed colour distribution for $414 G C$ candidates in NGC 4486 with galactocentric radii from 40 to 120 " and position angles between 30 and $150^{\circ}$. Dashed lines correspond to the TVP (see text).

Table 2. Mono-colour 12 Gy GC population fits in Virgo galaxies.

\begin{tabular}{cc}
\hline \hline Number of GC & {$[\mathbf{Z} / \mathbf{H}]$} \\
\hline 270 & -1.65 \\
257 & -1.31 \\
142 & -0.93 \\
143 & -0.67 \\
137 & -0.46 \\
143 & -0.30 \\
\hline
\end{tabular}

The adopted model, finally, is the mean of ten different realizations in an attempt to minimize the statistical noise of the models.

The result for the Virgo galaxies is shown in Fig. 28 and listed in Table 2. This case requires six discrete components. However, the fit suggests the presence of an extra component, at the reddest extreme of the distribution (shown in the residuals curve), but not detectable as peaks in the composite $G C C D$ of these galaxies. This red broad residual, in turn, overlaps with the position of two colour peaks included in the $T V P$.

The case of the GCs associated with the counterpart galaxies in Fornax is displayed in Fig. 29 and listed in Table 3 . For these galaxies the composite $G C$ colour distribution and the TFP shows a single broad blue peak. However, the decomposition procedure requires at least two blue components. The residuals curve shows that two more components (at the bluest and reddest extremes) would be required for a proper fit over the whole colour range. This indicates that, the broad and single blue peak [2] detectable in the Fornax galaxies that define the TFP, may arise as the

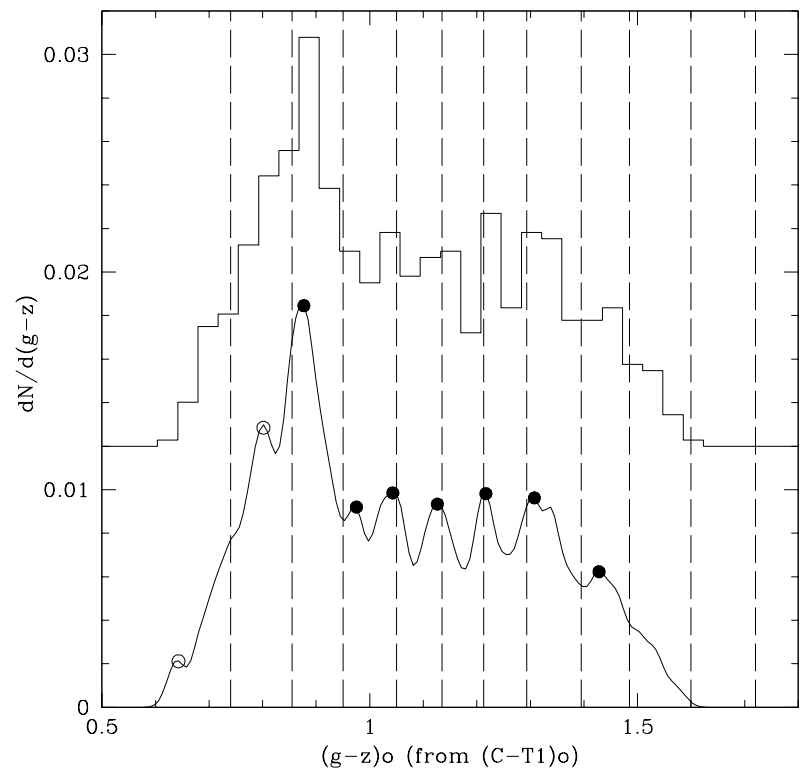

Figure 23. Discrete histogram (arbitrarily shifted upwards) and smoothed colour distribution for 683 GCs in $N G C 4486$ with galactocentric radii between 90 and $150 "$. Dashed lines correspond to the TVP (see text).

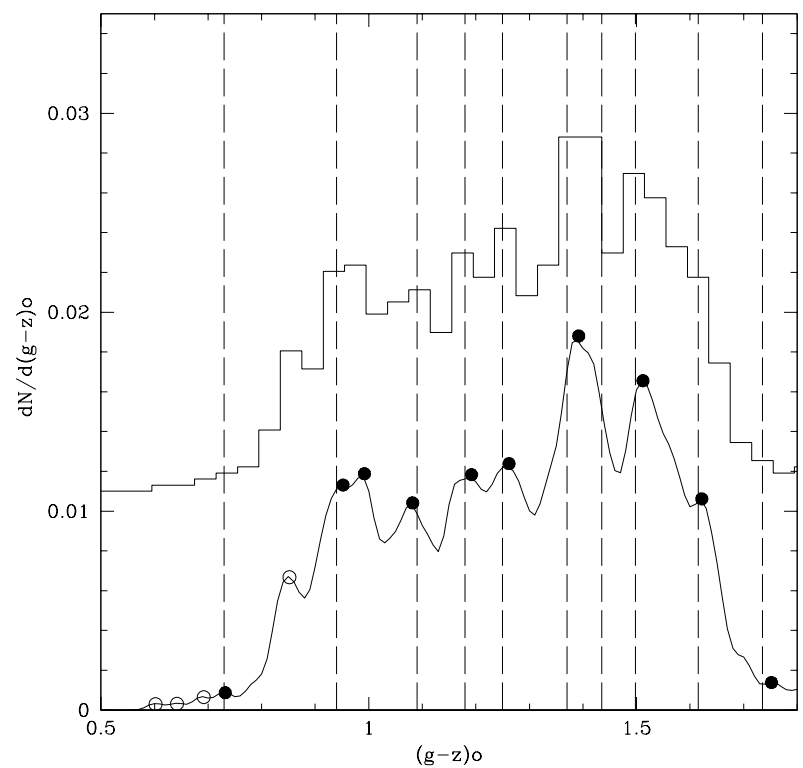

Figure 24. Discrete histogram (arbitrarily shifted upwards) and smoothed $(g-z)$ colour distribution for 814 GCs in NGC 1399 with galactocentric radii from 0 to 120 ".

result of two to three ("non-resolved") mono-colour components. The main conclusion of this Section is that, without discarding other possible approaches, the decomposition of the $G C C D s$ in terms of discrete mono-colours components cannot be rejected, at the level of the photometric errors, for galaxies with $M_{g}=-20.2$ to -19.2 both in Virgo and Fornax. 


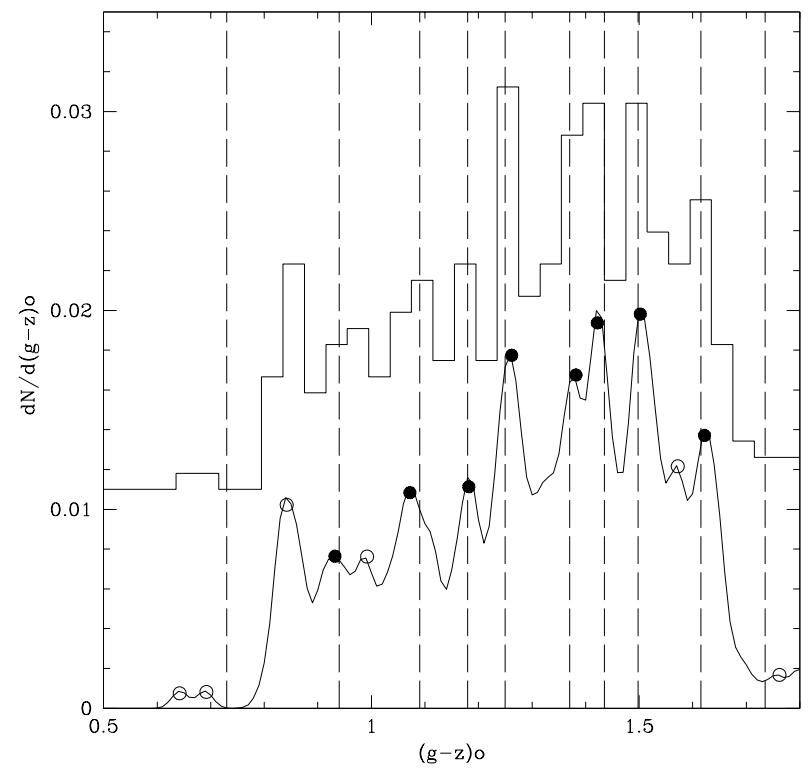

Figure 25. Discrete histogram (arbitrarily shifted upwards) and smoothed colour distribution for 309 GCs in NGC 1399 with galactocentric radii from 0 to 90 " and position angles between 90 and $270^{\circ}$. Dashed lines correspond to the $T F P$ (see text).

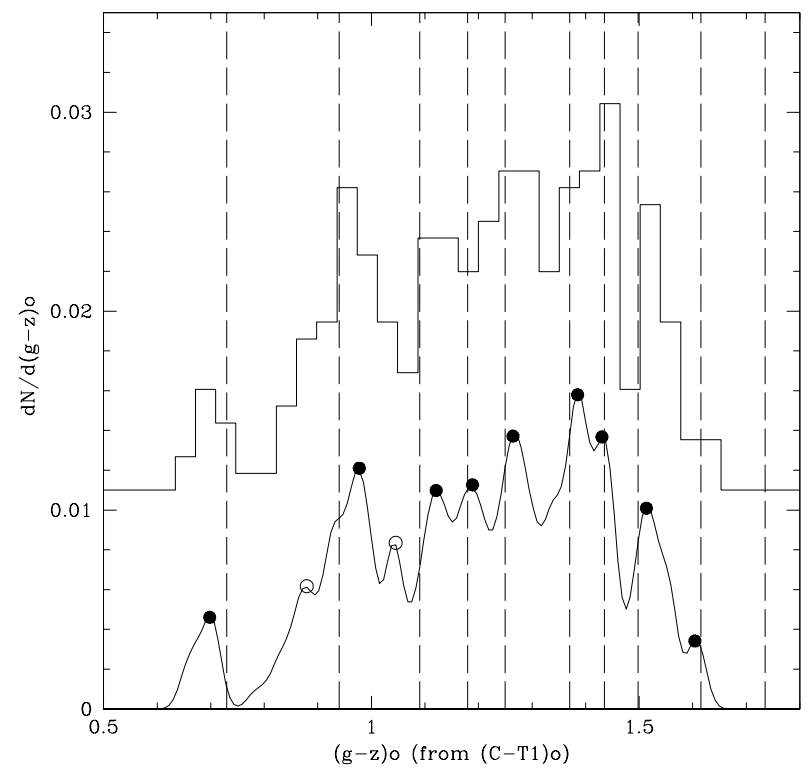

Figure 26. Discrete histogram (arbitrarily shifted upwards) and smoothed colour distribution for 293 GCs in NGC 1399 with galactocentric radii from 0 to 120 " and position angles between 90 and $360^{\circ}$. Dashed lines correspond to the TFP (see text).

\section{DISCUSSION}

The analysis presented in previous sections shows that colour modulation patterns are present in the GCCDs of composite $G C$ samples in some of the brightest Virgo and Fornax galaxies.

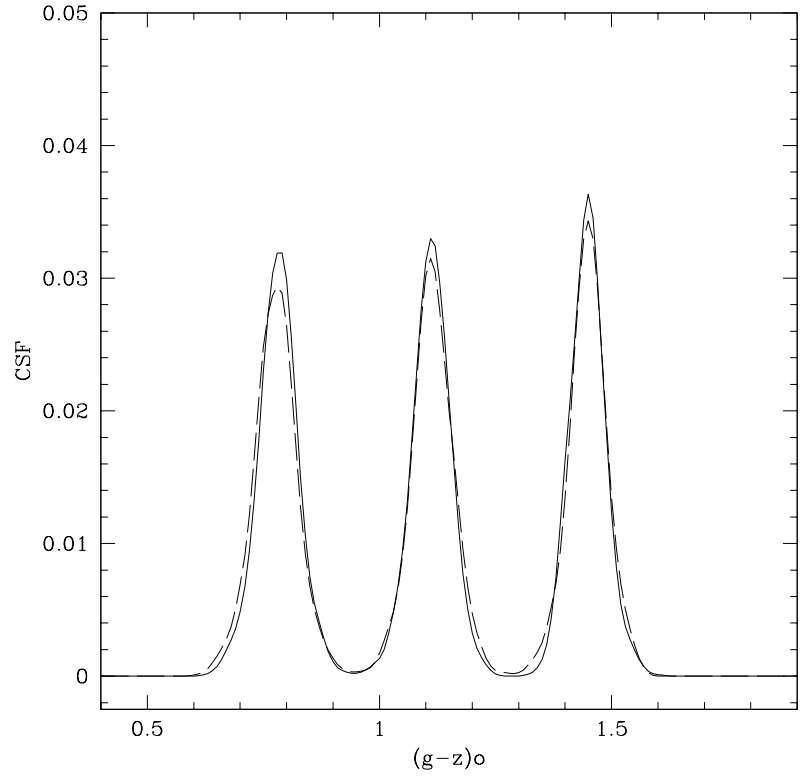

Figure 27. Colour spread function (CSF) for GCs in Fornax (dashed lines) and Virgo (continuous lines) corresponding to three chemical abundances (left to right: $[\mathrm{Z} / \mathrm{H}]=-2.0,-0.70$ and 0.00 ). These functions include the effect of photometric errors and are convolved with a Gaussian kernel of 0.015 mag in $(g-z)$.

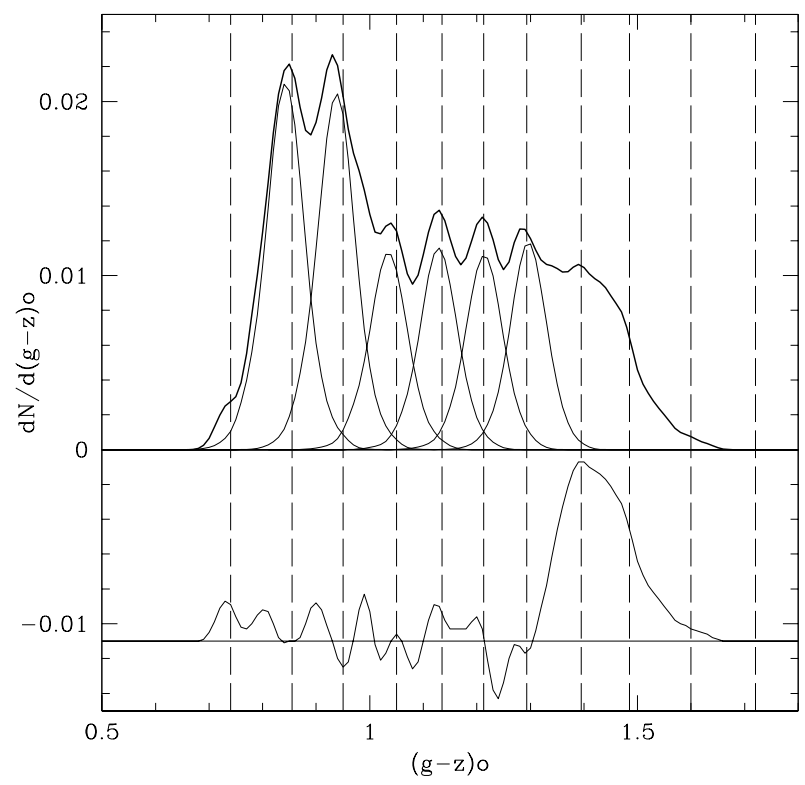

Figure 28. Smoothed colour distribution for GCs defining the template pattern in Virgo galaxies (upper curve). The six components of the CSF are shown individually (thin lines). The observed minus model residuals are displayed in the lower curve (shifted by -0.011 in ordinates). Vertical lines indicate the $T V P$ colours. A broad red feature in the residual curve may be decomposed in other two components present in the TVP. 
Table 3. Mono-colour 12 Gy GC population fits in Fornax galaxies.

\begin{tabular}{cc}
\hline \hline Number of GC & {$[\mathbf{Z} / \mathbf{H}]$} \\
\hline 181 & -1.41 \\
171 & -1.15 \\
130 & -0.80 \\
123 & -0.56 \\
117 & -0.38 \\
132 & -0.17 \\
\hline
\end{tabular}

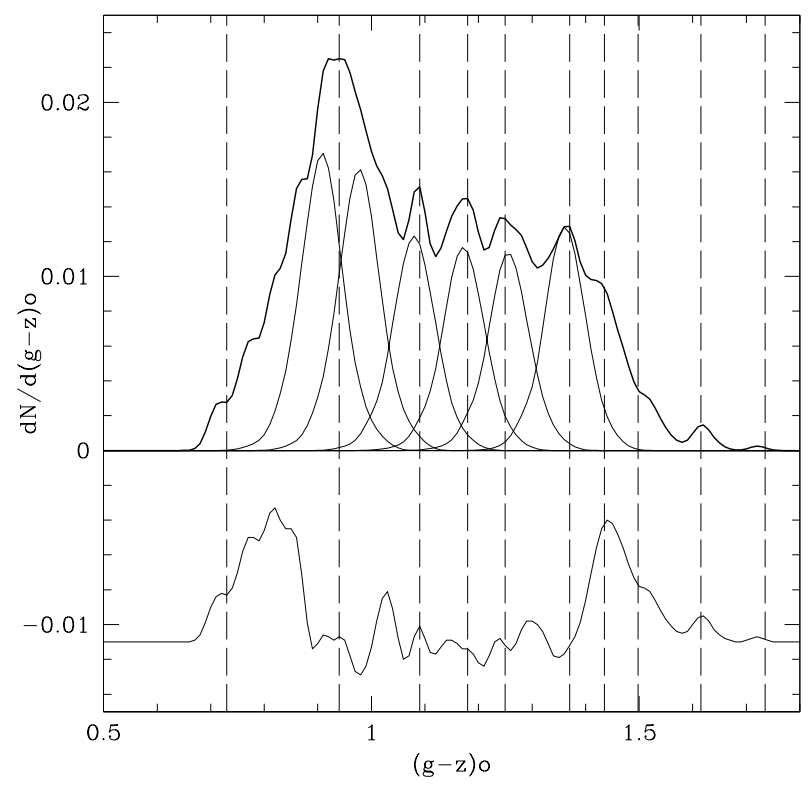

Figure 29. Smoothed colour distribution for GCs defining the template pattern in Fornax galaxies (upper curve). The six components of the CSF are shown individually (thin lines). The observed minus model residuals are displayed in the lower curve (shifted by -0.011 in ordinates). Vertical lines indicate the colours defined by the TFP (see text). The two broad features in the blue and red extremes suggest the presence of other two components.

In principle, these modulations may have different origins. Namely, field contamination, some kind of errors hidden in the photometric data, statistical sampling noise or, finally, an alternative possibility that assumes that the template patterns have a physical entity. In what follows we comment on each of these items.

\subsection{Possible origin of the modulation pattern}

\subsubsection{Field contamination}

On one side, the discussion of this issue given in Jordán et al. (2009) and Jordán et al. (2015), indicates that field contamination above the GCs magnitude cut off adopted in this work ( $g=25.0$ ) should be negligible. On the other, the effect of eventual field contaminants should be more important in the less populated composite GCCDs of the fainter galaxy groups. The results for these samples show that this is not the case, i.e., field contamination can be safely ruled out as tentative origin of the colour patterns.

\subsubsection{Photometric errors}

The appearance of the colour modulations might suggest the presence of some kind of "periodic" errors (in colour terms) hidden in the ACS photometric data. Such errors have not been reported in the literature. On the other side, the template patterns are also detectable on independent data sets (e.g. the ground based Washington photometry and the Gemini - GMOS photometry). Based on these results, the presence of eventual errors can be rejected.

\subsubsection{Statistical noise}

The histograms corresponding to composite $G C$ samples in Virgo and Fornax (for galaxies with $M_{g}=-20.2$ to -19.2 ) presented initially in Fig. 9 and Fig. 10 show differences and similarities. For example, Virgo galaxies exhibit a double blue peak $[2,3]$ while their Fornax counterparts show a single and broad blue peak [2]. In turn, both groups of galaxies present four to five colour peaks in the range occupied by intermediate-to-red GCs.

The amplitude of these modulations is comparable to the expected Poissonian noise corresponding to the mean population of intermediate-red GCs per bin. This argument might be considered as enough support in favour of statistical count fluctuations as the origin of the colour modulations.

Moreover, $G C$ populations modelling based on a Monte Carlo approach (e.g. Forte et al. 2007), can lead to statistical count fluctuations that, under given circumstances, show some similarities with the observed colour modulations. However, these models are unable to replicate the survivability of the colour patterns when adopting different seed parameters or when the $G C C D s$ of the artificial clusters are analysed in subgroups characterized by ranges in magnitude (as shown in Fig. A2 and Fig. A4).

An elementary statistical approach can be done assuming colour cells with sizes comparable to the $F W H M$ of the CSFs ( $\approx 0.1$ mag.) discussed in the previous Section. The most evident ( 6 to 7 ) colour peaks in the $G C C D s$ fall within a $(g-z)$ colour range of $0.8 \mathrm{mag}$ (0.8 to 1.6) then comprising eight of these cells. With these parameters, the combinatorial number leads to 8 different possible patterns (with 7 peaks),i.e., a particular one has a 12 percent probability of arising as the result of a random process. However, if the colour pattern can be recognized after spliting the $G C$ sample, the random probability is decreased to only 1.5 percent.

Furthermore, the distinct template colour patterns emerged from the composite GCCDs of galaxies within a given range of absolute magnitudes $\left(M_{g}=-20.2\right.$ to -19.2$)$ and were later found in the individual GCCDs in cluster subsamples in the most massive elliptical galaxies: NGC 4472 and NGC 4486 in Virgo, and NGC 1399 and NGC 1404 in Fornax. In fact the brightest galaxy in Fornax is NGC 1316 and, even in this complex system, some features of the TFP are detectable (see Fig. A8).

On the basis of all these arguments, stochastic effects can be rejected as the origin of the colour patterns. 


\subsubsection{An alternative possibility}

After rejecting the eventual possibilities described above, we assume that the colour patterns are real, distinct in Virgo and Fornax, and suggest the existence of a previously unknown feature common to $G C S$ in both galaxy clusters. A tentative explanation involves the effect of outer stimuli leading to the enhancement of the GCs formation process on different galaxies, in a synchronized way, as discussed below.

The main underlying questions are then, how such a features may emerge on supra-galactic scales, and why they can be detected more easily in galaxies with a given range in $M_{g}$ ?

Leaving the first question for the next sub-section, we can argue that galaxies in the magnitude range from $M_{g}$ -20.2 to -19.2 seem somewhat "privileged" systems for the detection of the template patterns: They have significant number of $G C s$ and, at the same time, have reached chemical enrichment levels as to include a considerable number of red GCs.

The discussion presented in the previous Section in fact suggests that outer stimuli (enhancing the formation of mono-colour cluster populations) may have contributed to an important fraction of the total $G C S$ in these galaxies.

Fainter and less massive galaxies reach lower chemical enrichment levels and would have not been able to show evident features in the domain of the red GCs (except a few cases noted previously (e.g. Fig. 19).

Invoking a "downsizing" scenario, most of these galaxies would be younger than the brighter and more massive counterparts, and formed at redshifts $z \approx 2$ or lower (see Paulino-Afonso et al. 2017), i.e., after the era when most of the energetic events took place (in the tentative time scale defined by $M W-G C s$ ).

In the case of $N G C 4486$ and $N G C$ 1399, and other massive galaxies, their rich $G C S$ presumably include clusters formed in a variety of processes that characterize their own histories. The colour patterns imposed by a putative external mechanism, would be overlapped in colour to those clusters but probably exhibiting differences in age. Usher et al. (2015) report that, in their analysis of the $G C$ colourmetallicity relations in different galaxies, these authors find a spread possibly compatible with differences in $G C$ ages.

If the colour patterns are the result of external events, a chronological scale could be set on the basis that chemical abundance $[Z / H]$ correlates with time. For example, Forbes et al. (2015) show that blue and red GCs exhibit substantial age differences, being the former about $1.5 \mathrm{~Gy}$ older. In an a priori way, the $G C C D$ statistics may reflect either a temporal sequence (where $[Z / H]$ is a "clock"), a positional sampling (i.e. halo, bulge), or a combination of both. This seems to be the case, for example, of $G C s$ in the $M W$.

These clusters show a bifurcated age- $[Z / H]$ relation corresponding to halo or bulge-disc GCs (see Leaman et al. 2013). Both branches of that relation exhibit similar slopes in the age- $[Z / H]$ plane and an offset of 0.6 dex in chemical abundance (in the sense that bulge-disc clusters have higher $[Z / H])$.

The existence of such relations in other galaxies is only an arguable guess and some differences can be expected. For example, more massive galaxies may display shallower slopes (eventually due to faster chemical enrichment) then

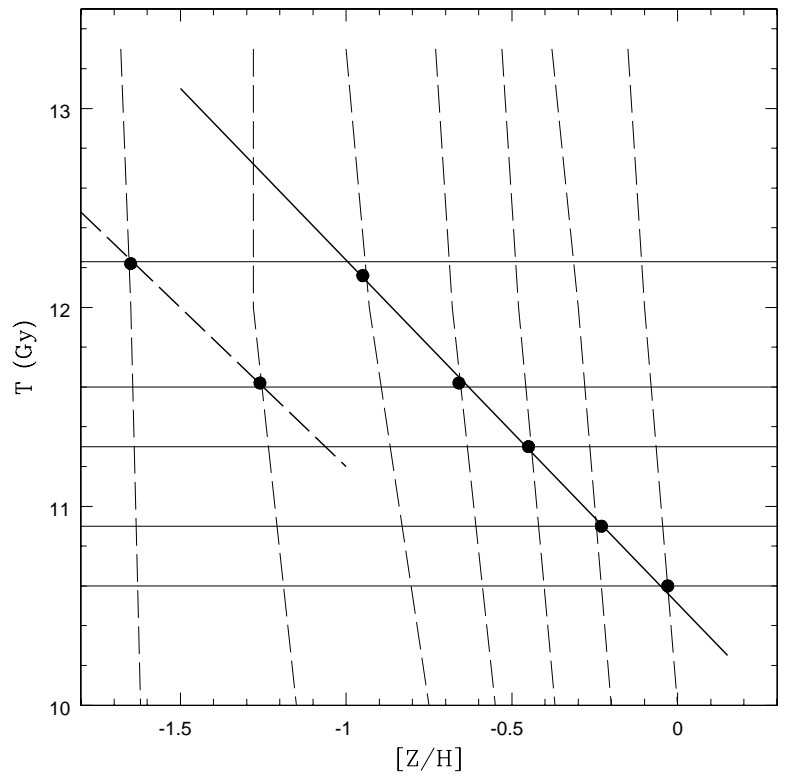

Figure 30. The bifurcated age-metallicity relation for halo (thick dashed line) and bulge-disk (thick solid line) GCs in the $M W$. Thin dashed lines represent constant model $(g-z)_{o}$ colours corresponding to seven of the colour peaks observed in Virgo GCs $([2,3,4,5,6,7,8] ;$ see text). The interception of the age-metallicity relations with the constant colour lines indicate the age of eventual external events triggering $G C$ formation (horizontal lines). The most recent events (filled dots) would impact only on the bulge-disc $G C s$. The events span a range of $\approx 1.4 \mathrm{~Gy}$.

compressing the time scale. This is an important and obvious caveat in the following analysis.

The bifurcated age- $[Z / H]$ relations for $M W$ clusters are shown as thick dashed lines (blue halo GCs) and thick solid lines (bulge-disc GCs in Fig. 30 and Fig. 31. Thin dashed lines in these diagrams correspond to the most prominent features in the TVP $[2,3,4,5,6,7,8]$ and TFP $[2,3,4,5,6]$ patterns listed in Table 2.

The constant colour lines were derived from SSP models by Bressan et al. (2012) and include a small correction to the $g$ magnitudes (that depend on metallicity) as described in Sesto et al. (2016).

The interception between the $M W$ age- $[Z / H]$ relations and these lines would indicate the age (thin horizontal lines) as well as the level of chemical enrichment of the interstellar medium in a galaxy at the time of a given event leading to the enhancement of the $G C$ formation. The "width" of each of the colour features that define the template patterns is in fact dominated by photometric errors (as dicussed in Section 7). This is a limiting factor for an estimate of the time lapse involved in each of these events.

Fig. 30 and Fig. 31 also show that the two first events would have had a simultaneous impact on both $G C$ metallicity sequences, eventually leading to the formation of two peaks separated in colour.

The time lapse of these events range from $\approx 0.2$ to 0.6 Gy. A comparison between Fig. 30 and Fig. 31 indicates that the earliest events in Virgo would have taken place some 0.4 Gy earlier than in Fornax. 


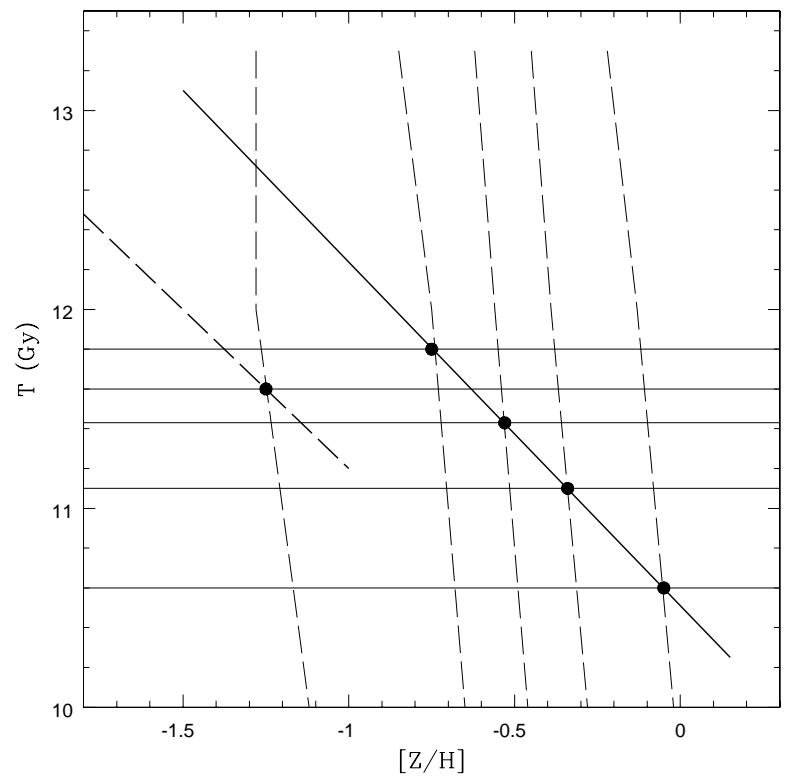

Figure 31. The bifurcated age-metallicity relation for halo (thick dashed line) and bulge-disk (thick solid line) $G C s$ in the $M W$. Thin dashed lines represent constant model $(g-z)_{o}$ colours corresponding to five of the colour peaks observed in Fornax $G C s$ $([2,3,4,5,6] ;$ see text). The interception of the age-metallicity relations with the constant colour lines indicate the age of (eventual) external events triggering $G C$ formation (horizontal lines). The most recent events (filled dots) would impact only on the bulgedisc GCs. The events span a range in time of $\approx 1.2$ Gy.

\subsection{Possible origin of the mono-colour GC populations}

\subsubsection{Super Massive Black Holes}

The role of Super Massive Black Holes ( $S M B H s$ ) in this context remains to be clarified. Interestingly, Harris, Poole \& Harris (2014) find a strong correlation between the dynamical mass of galaxy bulges and the number of associated clusters and stress that a causal $G C s-S M B H$ link cannot be simply dismissed. Both Virgo and Fornax have a number $S M B H$ (see Table 1 in the Harris et al. work) that might have been potential players in generating high energy outflows producing an impact on the star forming process and, in particular, on the $G C$ formation.

\subsubsection{Cluster-Cluster mergers}

A number of other possible mechanisms deserve to be explored. For example, in $\Lambda$-CDM cosmologies, galaxy clusters are the result of the merger of smaller sub-clusters. An outstanding object in this frame is CIZAJ22428+5301 (Sobral et al. 2015; Stroe et al. 2015), an old galaxy cluster at $z=0.19$, with a violent stellar formation activity fuelled by a galaxy cluster merger. This activity takes place over a diameter of $\approx 3 M p c$ a spatial scale that is considerably larger than, for example, the core radii of the Virgo and Fornax clusters (in the order of $\approx 1 \mathrm{Mpc}$ ).

A possible scenario suggests that, the strong shock waves originated in sub-cluster mergers at high red-shifts, may have favoured the formation of $G C s$ if the proper sites (dark matter mini-haloes ?) are available in a galaxy. The eventual availability of these places might explain the lack of a clear regularity in the amplitude of the colour patterns in different $G C S$.

\section{CONCLUSIONS}

The nature of the distinct colour modulations in the GCCDs of clusters associated with Virgo and Fornax galaxies, and characterized through the so called "template patterns" ( $T V P$ and $T F P$ ), is an intriguing question. These structures are no evident in currently available spectroscopic data, possibly as a result of the still relatively large errors in age and chemical abundance.

At this point, the main argument to support their entity as features with a physical meaning, stands on the fact that, once recognized in a given sub-group of galaxies that defined the template colour patterns, they can be found also in the rich GCS of the central galaxies NGC 4486 and NGC 1399, as well as in other bright galaxies (not included in the galaxy groups that define the template patterns).

The GCS associated with these massive galaxies reflect their own and complex histories including, for example, the accretion of GCs belonging to less massive systems during mergers (Forte, Martinez \& Muzzio 1982; Côté, Marzke \& West 1998). We note that, in these processes, dry mergers involving galaxies that already have incorporated the template pattern structures, would preserve these features.

The physical origin of these colour patterns, shared by a number of galaxies spread on a large spatial volume, at this stage is clearly speculative. However, their detection has been the result of the motivation of this work given in the Introduction: The search for detectable signatures, possibly connected with energetic events, on the GCs formation process.

An important and open issue connected with GCs is why they do not form currently (except, possibly, in on-going galaxy mergers) despite that enough interstellar matter is available to feed such a process (for example, in the $M W$ ). The missing ingredient can be (cautiously) identified with those events.

The spatial positions of the galaxies that define the template colour patterns do not show an evident systematic distribution, i.e., these galaxies are spread over the whole Virgo and Fornax clusters. The positions of these galaxies, however, do not necessarily reflect the conditions prevailing at the high red-shift regime when the energetic events eventually took place.

In any case, it is clear that these phenomena should have been powerful enough to leave an imprint on large spatial volumes and possibly not only on GCS but also on the galaxy stellar populations in general. For example, ultra compact dwarfs (UCDs) include distinct stellar sub populations with rather defined ages and chemical abundances (e.g. Norris et al. 2015) that may also fit in that landscape.

Rather than stellar formation "quenching" the (peaked) colour patterns suggest that the putative phenomenon has been able to enhance the formation of GCs . Several mech- 
anisms that may increase the star formation rate have been proposed in the recent literature (Nayakshin \& Zubovas 2012; Silk 2013; Abe, Umemura \& Hasegawa 2016)

The similarities of the colour patterns in Virgo and Fornax might be related to environmental conditions (i.e., these are galaxy clusters with dominant giant galaxies).

The detectability of these features in other environments, e.g., the Local Group, with much smaller $G C$ populations will be presumably hard to asses. However, it is possible that large scale events may leave an imprint not only on the $G C C D s$ but also on individual $G C s$.

For example, the presence of multi populations in $M W$ clusters, as the remarkable case of Terzan 5 (see Ferraro et al. 2016), may provide some clues about the eventual impact of those phenomena on the life of galaxies located in poorly populated environments.

Finally, as an unavoidable conclusion, the analysis of the $G C s$ colour patterns will require a considerable volume of high precision photometry and spectroscopy (involving very low errors both in age and $[Z / H]$ ) for a complete characterization of their astrophysical properties.

\section{ACKNOWLEDGEMENTS}

This work was funded with grants from Consejo Nacional de Investigaciones Cientificas y Tecnicas de la Republica Argentina (CONICET). Thanks are also due to Dr. E. Irene Vega for her continuous support during the redaction of this work.

\section{REFERENCES}

Abe M., Umemura M., Hasegawa K., 2016, MNRAS, 463, 2849 Bellini A., et al., 2015, ApJ, 805, 178

Binggeli B., Sandage A., Tarenghi M., 1984, AJ, 89, 64

Blakeslee J. P., et al., 2009, ApJ, 694, 556

Bressan A., Marigo P., Girardi L., Salasnich B., Dal Cero C., Rubele S., Nanni A., 2012, MNRAS, 427, 127

Brodie J. P., Strader J., 2006, ARA\&A, 44, 193

Cen R., 2001, ApJ, 560, 592

Chen C.-W., Côté P., West A. A., Peng E. W., Ferrarese L., 2010, ApJS, 191, 1

Chonis T. S., Gaskell C. M., 2008, AJ, 135, 264

Côté P., Marzke R. O., West M. J., 1998, ApJ, 501, 554

D'Abrusco R., Fabbiano G., Zezas A., 2015, ApJ, 805, 26

Fabian A. C., 2012, ARA\&A, 50, 455

Faifer F. R., et al., 2011, MNRAS, 416, 155

Ferguson H. C., 1989, AJ, 98, 367

Ferraro F. R., Massari D., Dalessandro E., Lanzoni B., Origlia L., Rich R. M., Mucciarelli A., 2016, ApJ, 828, 75

Forbes D. A., Ponman T., O'Sullivan E., 2012, MNRAS, 425, 66

Forbes D. A., Pastorello N., Romanowsky A. J., Usher C., Brodie J. P., Strader J., 2015, MNRAS, 452, 1045

Forbes D. A., Alabi A., Romanowsky A. J., Brodie J. P., Strader J., Usher C., Pota V., 2016, MNRAS, 458, L44

Forte J. C., Martinez R. E., Muzzio J. C., 1982, AJ, 87, 1465

Forte J. C., Faifer F., Geisler D., 2007, MNRAS, 382, 1947

Forte J. C., Vega E. I., Faifer F., 2012, MNRAS, 421, 635

Forte J. C., Faifer F. R., Vega E. I., Bassino L. P., Smith Castelli A. V., Cellone S. A., Geisler D., 2013, MNRAS, 431, 1405

Forte J. C., Vega E. I., Faifer F. R., Smith Castelli A. V., Escudero C., González N. M., Sesto L., 2014, MNRAS, 441, 1391
Goudfrooij P., Mack J., Kissler-Patig M., Meylan G., Minniti D., 2001, MNRAS, 322, 643

Harris G. L. H., Poole G. B., Harris W. E., 2014, MNRAS, 438,2117

Harris W. E., Ciccone S. M., Eadie G. M., Gnedin O. Y., Geisler D., Rothberg B., Bailin J., 2017, ApJ, 835, 101

Jordán A., et al., 2009, ApJS, 180, 54

Jordán A., Peng E. W., Blakeslee J. P., Côté P., Eyheramendy S., Ferrarese L., 2015, ApJS, 221, 13

Leaman R., VandenBerg D. A., Mendel J. T., 2013, MNRAS, 436, 122

Milone A. P., et al., 2017, MNRAS, 464, 3636

Nayakshin S., Zubovas K., 2012, MNRAS, 427, 372

Norris M. A., Escudero C. G., Faifer F. R., Kannappan S. J., Forte J. C., van den Bosch R. C. E., 2015, MNRAS, 451, 3615

Paulino-Afonso A., Sobral D., Buitrago F., Afonso J., 2017, MNRAS, 465, 2717

Peng E. W., et al., 2006, ApJ, 639, 838

Richtler T., Bassino L. P., Dirsch B., Kumar B., 2012, A\&A, 543, A131

Schlafly E. F., Finkbeiner D. P., 2011, ApJ, 737, 103

Sesto L. A., Faifer F. R., Forte J. C., 2016, MNRAS, 461, 4260

Silk J., 2013, ApJ, 772, 112

Sobral D., Stroe A., Dawson W. A., Wittman D., Jee M. J., Röttgering H., van Weeren R. J., Brüggen M., 2015, MNRAS, 450,630

Stroe A., et al., 2015, MNRAS, 450, 646

Usher C., et al., 2015, MNRAS, 446, 369

VanDalfsen M. L., Harris W. E., 2004, AJ, 127, 368

Vanzella E., et al., 2016, preprint, (arXiv:1612.01526)

Villegas D., et al., 2010, ApJ, 717, 603

Vogelsberger M., et al., 2014, MNRAS, 444, 1518

\section{APPENDIX A: ADDITIONAL DIAGRAMS.}




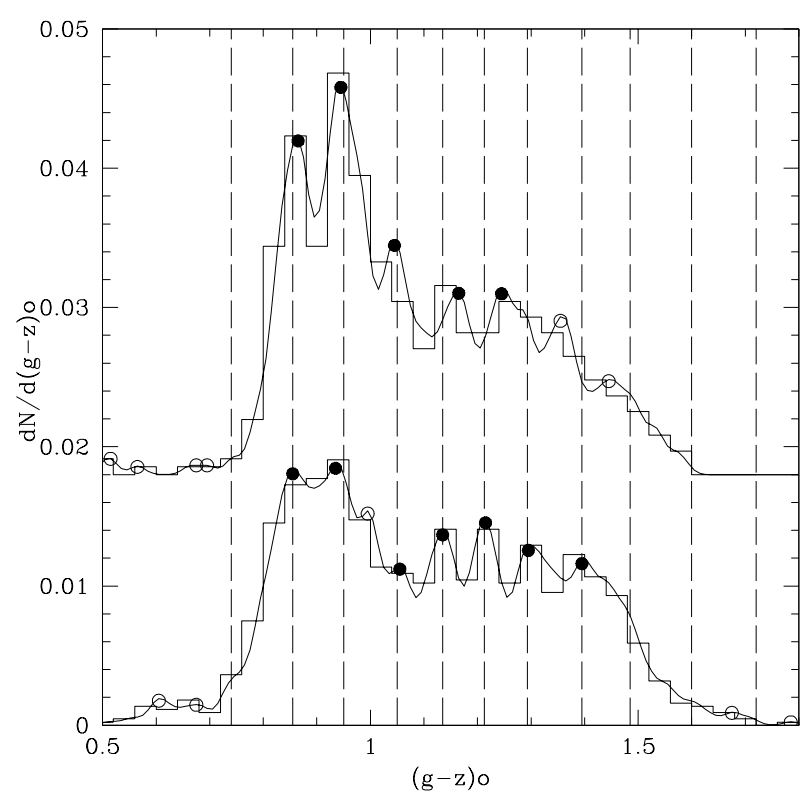

Figure A1. Discrete and smoothed (g-z)o colour distribution for $G C s$ in 13 Virgo galaxies with $M_{g}$ between -20.2 and -19.2. The histogram have $0.04 \mathrm{mag}$ bins while the smoothed distributions correspond to a 0.015 mag Gaussian kernel. The upper histogram (arbitrarily shifted upwards) includes 437 GCs with apparent magnitude $g$ from 20 to 23. The lower histogram includes 1098 GCs with $g$ from 23.0 to 25.0.

This paper has been typeset from a $\mathrm{T}_{\mathrm{E}} \mathrm{X} / \mathrm{LAT}_{\mathrm{E}} \mathrm{X}$ file prepared by the author.

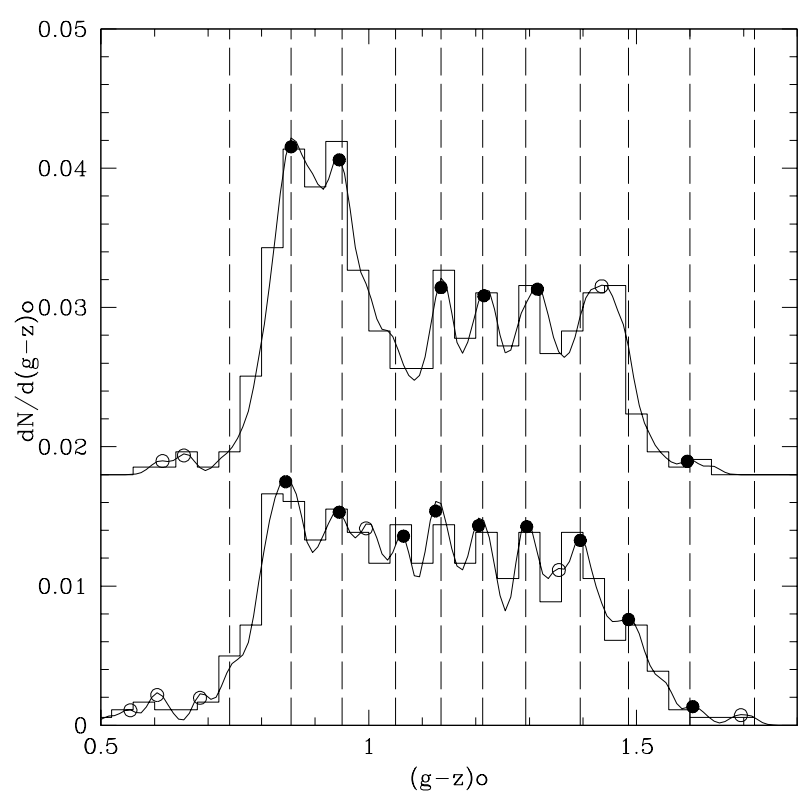

Figure A2. Discrete and smoothed (g-z)o colour distribution for $G C s$ in 13 Virgo galaxies with $M_{g}$ between -20.2 and -19.2. The upper histogram (arbitrarily shifted upwards) corresponds to $G C s$ with $g=23.0$ to 23.75 and includes 458 clusters; the lower one corresponds to $450 \mathrm{GCs}$ with $g=23.75$ to 24.50 .

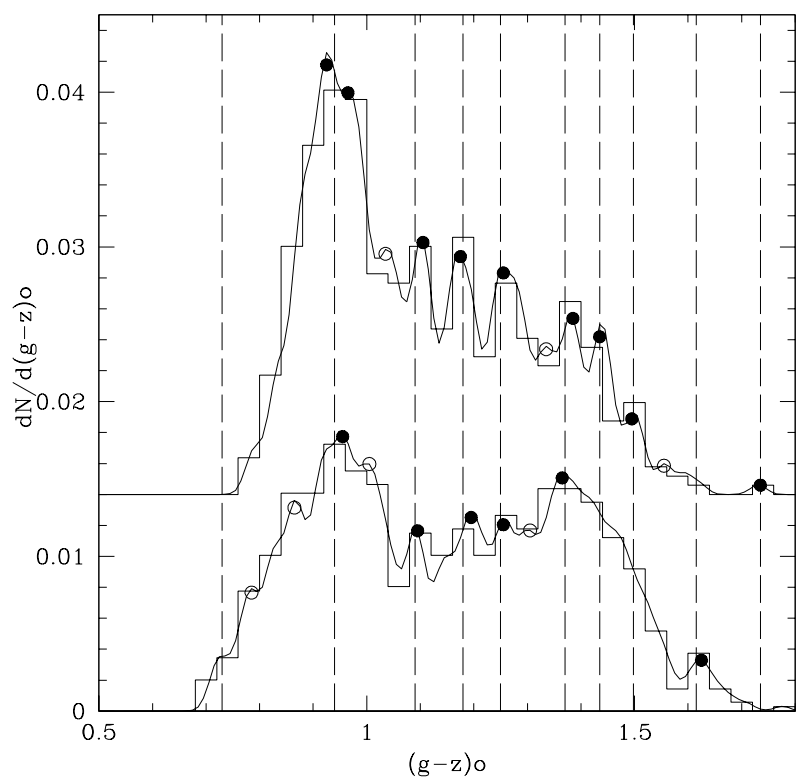

Figure A3. Discrete and smoothed (g-z)o colour distribution for $G C s$ in 7 Fornax galaxies with $M_{g}$ between -20.2 and -19.2 . The histograms have $0.04 \mathrm{mag}$ bins while the smoothed distributions corresponds to a 0.015 mag Gaussian kernel. The upper histogram (arbitrarily shifted upwards) includes 417 GCs with apparent magnitude $g$ from 20.0 to 23.4. The lower histogram corresponds to $852 G C s$ with $g$ from 23.4 to 25.0. 


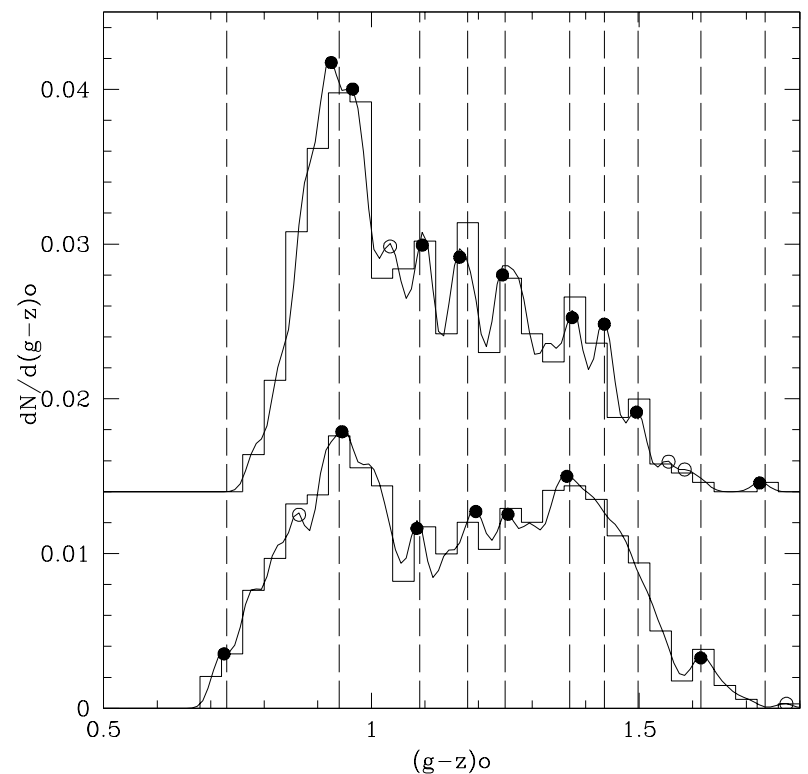

Figure A4. Discrete and smoothed $(g-z) o$ colour distribution for GCs in 7 Fornax galaxies with $M_{g}$ between -20.2 and -19.2. The upper histogram (arbitrarily shifted upwards) includes 417 GCs with $g=20.0$ to 23.4 ; the lower one corresponds to 413 GCs with $g=23.40$ to 24.20 .

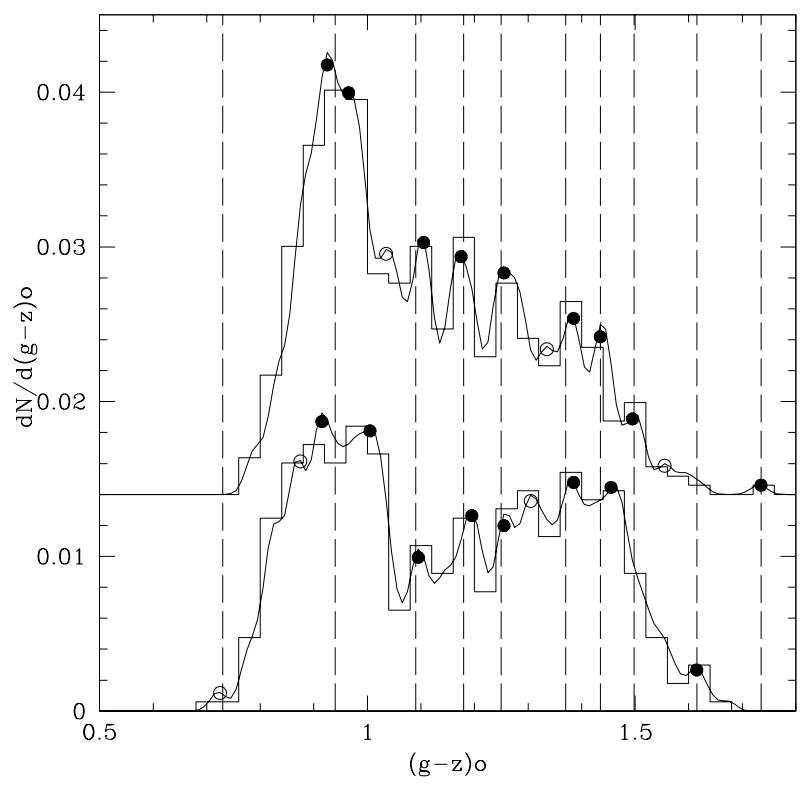

Figure A5. Discrete and smoothed $(g-z) o$ colour distribution for 522 GCs candidates in an offset field in NGC 4486 and observed with Gemini-GMOS (upper histogram, arbitrarily shifted upwards), compared with that of GCs in Virgo galaxies with $M_{g}=-20.2$ to -19.2 (lower curve) and with the TVP (dashed lines, see text).

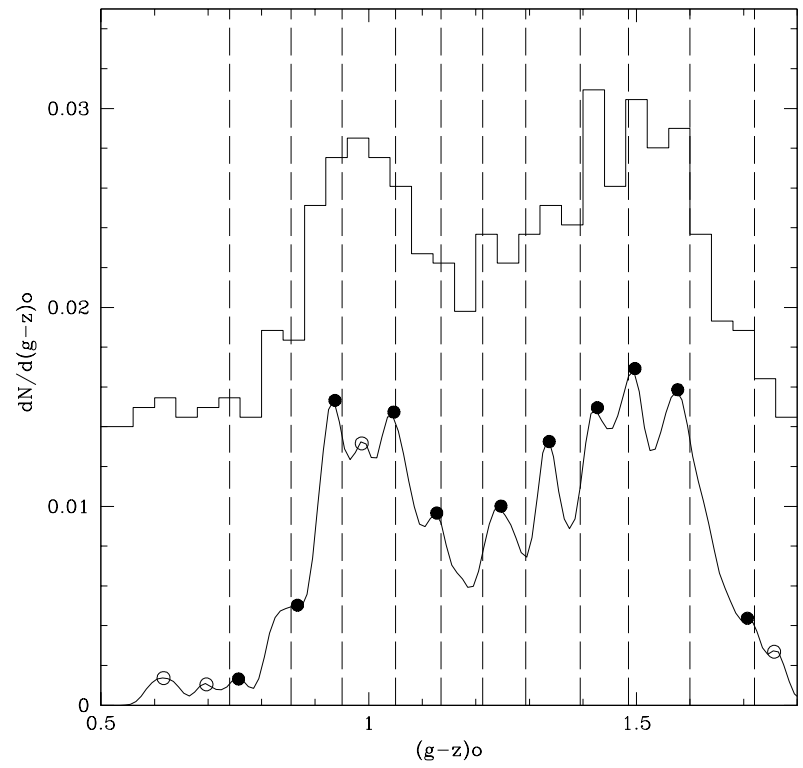

Figure A6. Discrete histogram (arbitrarily shifted upwards) and smoothed $(g-z) o$ colour distribution for 517 GCs in $N G C 4472$. The clusters have galactocentric radii between 20 and $110 "$. This $G C s$ sample exhibits the double blue peak seen in the $T V P$ and seven coincidences $[4,5,6,7,8,9,10$ and possibly 11$]$ in the domain of the intermediate and red GCs.

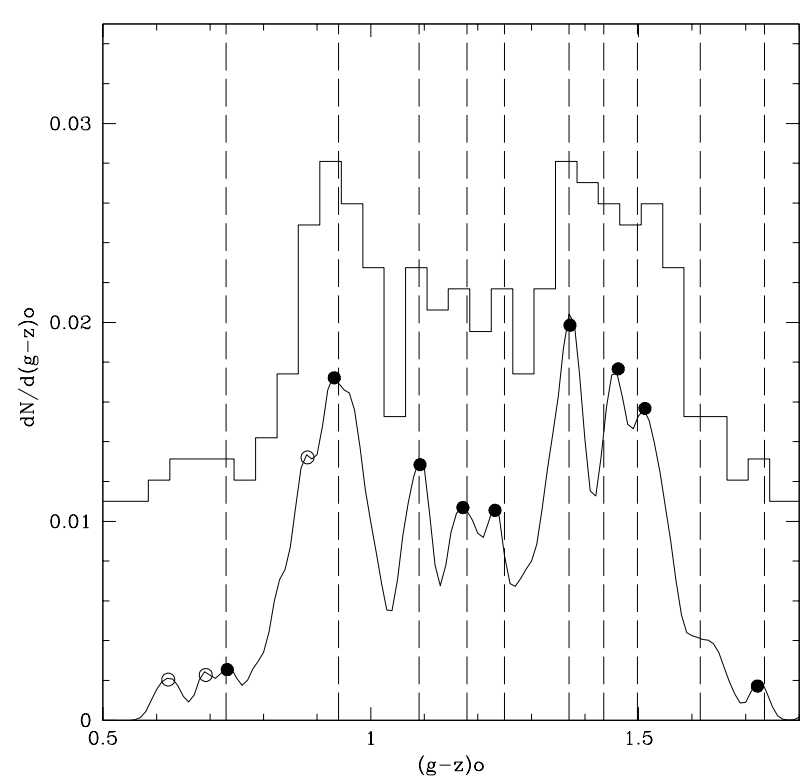

Figure A7. Discrete histogram (arbitrarily shifted upwards) and smoothed $(g-z) o$ colour distribution for 234 GCs in $N G C 1404$. The clusters have galactocentric radii between 0 and 80 ". 


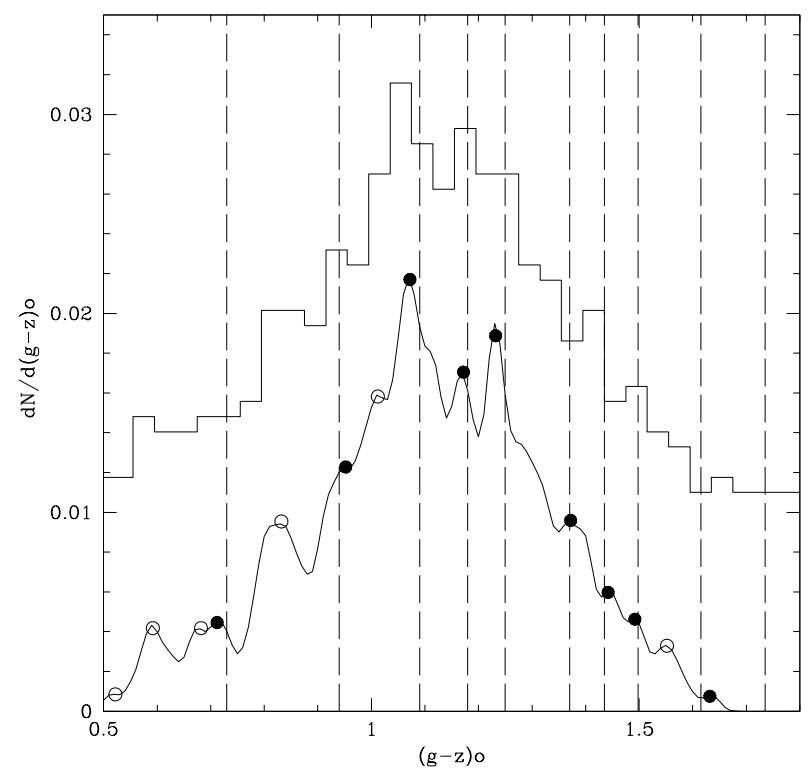

Figure A8. Discrete histogram (arbitrarily shifted upwards) and and smoothed $(g-z) o$ colour distribution for $367 \mathrm{GCs}$ with galactocentric radii from 40 to 120 " in NGC 1316. Several colour peaks on top of a broad $G C s$ colour distribution are coincident with those defined by the TFP. This colour distribution shows an extended blue tail arising in younger cluster populations (see text). 